\title{
Mathematical modelling of hepatic lipid metabolism
}

\author{
Adrian C Pratt ${ }^{\mathrm{a}, \mathrm{b}}$, Jonathan AD Wattis ${ }^{\mathrm{b}}$, Andrew M Salter ${ }^{\mathrm{a}}$ \\ ${ }^{a}$ Division of Nutritional Sciences, School of Biosciences, University of Nottingham, Sutton \\ Bonington Campus, Loughborough, Leicestershire, LE12 5RD, UK \\ ${ }^{b}$ Centre for Mathematical Medicine and Biology, School of Mathematical Sciences, University of \\ Nottingham, University Park, Nottingham, NG7 2RD, UK
}

\begin{abstract}
The aim of this paper is to develop a mathematical model capable of simulating the metabolic response to a variety of mixed meals in fed and fasted conditions with particular emphasis placed on the hepatic triglyceride element of the model. Model validation is carried out using experimental data for the ingestion of three mixed composition meals over a 24-hour period. Comparison with experimental data suggests the model predicts key plasma lipids accurately given a prescribed insulin profile. One counter-intuitive observation to arise from simulations is that liver triglyceride initially decreases when a high fat meal is ingested, a phenomenon potentially explained by the carbohydrate portion of the meal raising plasma insulin.
\end{abstract}

Keywords: fat metabolism, carbohydrate metabolism, mathematical models. 2000 MSC: 92C42 Systems biology, 92C45 Kinetics in biochemical problems.

Abbreviations: ACoA - Acetyl Coenzyme A, AMP - Adenosine Monophosphate, ATP - Adenosine Triphosphate, CHO - Carbohydrates, CM - Chylomicrons, DNL - De novo lipogenesis, FFA - Free Fatty Acids, G-3-P - Glycerol-3-Phosphate, G-6-P - Glucose6-Phosphate, LP - Lipoproteins, LPL - Lipoprotein Lipase, NAFLD - Non-alcoholic Fatty Liver Disease, NASH - Non-alcoholic Steatohepatitis, NEFA - Non-esterified Fatty Acids, TAG - Triacylglycerol/Triglycerides, TCA - Tricarboxylic acid cycle

Email addresses: stxap@nottingham.ac.uk (Adrian C Pratt),

Jonathan.Wattis@nottingham.ac.uk (Jonathan AD Wattis), Andrew.Salter@nottingham.ac.uk (Andrew M Salter) 


\section{Introduction}

Non-alcoholic fatty liver disease (NAFLD) is the chronic build up of excess lipids in the liver. NAFLD spans simple hepatic steatosis (HS) to hepatic steatosis accompanied by inflammation, termed non-alcoholic steatohepatitis (NASH), a condition that may progress to fibrosis and cirrhosis of the liver. Angulo \& Lindor [6] report the prevalence of NAFLD varies with estimates of 10-24\% of the populations in developed nations being normal. Of these individuals, $25 \%$ may progress to the more serious NASH. Prevalence of NAFLD amongst the obese is significantly higher (75\%), as is the prevalence in individuals with type 2 diabetes. With an increasing number of cases of NAFLD being diagnosed, a better understanding of the pathology of hepatic steatosis is required.

A number of pathways interact directly in the deposition and removal of liver lipids. Beta-oxidation of fatty acids, for use as a fuel source within the liver, represents a significant contribution to the disposal of hepatic lipids. Whilst hepatic oxidation of fatty acids is unchanged between subjects with high and low liver fat 34], a difference between obese and lean individuals has been observed by Hodson et al [24]. It was found that obese individuals had increased hepatic fatty acid oxidation over lean individuals in the postprandial period, potentially explained by a reduced suppression of beta-oxidation due to hepatic insulin resistance. Synthesis and secretion of very low-density lipoproteins (VLDL) provide a means for the liver to remove excess triglycerides (TAG) from cytosolic stores. This process is heavily influenced by insulin with both the number and size of particles secreted into the blood affected. Once much of the TAG has been removed from plasma VLDL by other tissues, the remnant particles, low density lipoproteins (LDL), are returned to the liver. Similarly, intestinally-derived chylomicrons delivering lipids from the gut have most of their TAG removed before the remnant particle is taken up by the liver. The direct contribution from chylomicron remnants to liver fat is estimated to be in the region of $7.2 \%$ of total liver fat sources [14]. Ordinarily, de novo lipogenesis (DNL) represents a pathway for the disposal of large volumes of carbohydrates through the conversion to fatty acids within the liver. A typical fed state contribution to total liver fat has been estimated to be $23 \%$ [57], whilst fasted contributions are substantially lower at $4.7 \%$. 
However, individuals with NAFLD experience elevated DNL contributions to liver fat in the fasted state (26\% 14]) indicating a chronic up-regulation of this pathway. The largest contribution to hepatic lipid stores is from the lipolysis of visceral and subcutaneous adipose tissue, measured at $61.7 \%$ and $81.7 \%$ for healthy subjects in the fed and fasted states respectively [14].

Central to the debate on the pathology of hepatic steatosis is the role of insulin resistance. Insulin resistance in adipose tissue results in a failure to suppress lipolysis and therefore higher levels of non-esterified fatty acids (NEFA) in the blood. As the largest contributor to liver fat is NEFA, it is easy to see why insulin resistance may lead to a higher hepatic TAG concentration. Conversely, increased lipid deposits in the liver can cause insulin resistance through impaired liver function [49]. In general, fat stored in tissues other than adipose has the potential to cause insulin resistance through impaired function of these other tissues (notable examples are pancreatic insulin-secreting beta cells, and skeletal muscle). This interrelation between lipid metabolism and insulin resistance leads us to consider both lipid and carbohydrate metabolism in the study of hepatic steatosis.

Many mathematical models concerning metabolism already exist, focusing on a range of scales and areas pertinent to the study of hepatic lipid metabolism. Stable isotope tracers have been used in conjunction with compartmental modelling to elucidate the kinetics of apoB (a key structural protein in lipoproteins) and triglyceride across VLDL subfractions simultaneously. Adiels' model [2] has proven to be a particularly useful tool in determining how physiological features impact VLDL size and dyslipidemia [4, 3, 39, 38]. A more in-depth model of VLDL assembly has been proposed by Shorten and Upreti [50] in which the lipid composition of secreted VLDLs can be determined from uptake of individual free fatty acids after elongation and desaturation by liver enzymes. Another area of mathematical modelling is the hepatic uptake and metabolism of lipoproteins, and the competition between subclasses within this process [46, 58].

Initial modelling of in vivo metabolic systems focused on predicting the glucose and insulin response to carbohydrate loads, with applications in the study of diabetes. 
Bergman's minimal model [7] provided a method to ascertain insulin sensitivity and glucose effectiveness in response to oral glucose tolerance tests (OGTT). Since then, many models have expanded [13] and extended [54] this to other physiological situations. Increased understanding of insulin production has led to enhanced models of secretion [42], and the use of modelling combined with stable isotopes tracers has allowed for a better understanding of glucose rate of appearance in the blood, independent of other processes [40].

Several models exist for the study of metabolism in individual tissues, e.g. in skeletal muscle [36, 12], in adipose tissue [33, 31] and in the brain [45]. These models tend to rely on flux balance analysis to obtain information about large scale metabolic networks at steady state, and are thus not always suitable for studying postprandial dynamics. Previous work on whole body models of metabolism has included some, but not all, of the features we would like to include in a model focused on hepatic lipid metabolism. Jelic et al [27] produce a model focusing on NEFA dynamics which, from prescribed insulin profiles of an OGTT, accurately predict true NEFA concentrations. Man et al [41] consider muscle and adipose tissue together as peripheral tissues, along with liver and pancreatic beta cells, but do not include a fat element to their model. In a $\mathrm{PhD}$ thesis on the subject, Kim [31] produces a detailed model of the metabolic networks in multiple tissues and organs through flux balance analysis. Here, the focus is on the fasted state, with applications to exercise, not the long term dynamics.

Our aim in this paper is to develop a mathematical model capable of simulating changes in the concentration of hepatic fat. Thus our model must be capable of reproducing the metabolic response to mixed meals in both fed and fasted conditions. From our discussion of the pathways and metabolic abnormalities which may lead to hepatic steatosis, we take an integrated approach modelling other key tissues and metabolites in addition to liver triglyceride. These do not need to be modelled precisely, but rather retain enough information to allow flexibility in model assessment. 


\section{Mathematical modelling}

In the derivation below we introduce variables corresponding to the evolution of various species over time. We use $G$ to represent glucose, $P$ for glucose-6-phosphate, $R$ for pyruvate, $Y$ for glucogen, $A$ for FFA/NEFA, $L$ for glycerol, and $T$ (and $S$ ) for TAG concentrations. We use subscripts to distinguish between the several tissue types that we are concerned with: namely $m$ for muscle, $L$ for liver, $A$ for adipose tissue and $B$ for blood plasma, though in this last case, we use additional subscripts to make a distinction between, exogeneous and endogenous TAG, for example. Due to the large number of parameters used, it is impossible to have a consistent naming policy here, we have used combinations of $k_{*}, v_{*}, \alpha_{*}$ with subscripts that contain some information on the process being modelled. The kinetic equations are generally derived using the law of mass action, with complicating factors introduced where this simplest approach fails and a more complicated description is required. For example, MichaelisMenten rate kinetics [43] are used in some places, where the effects of other species acting as catalysts or inhibitors is significant. In a couple of places, this is extended further, to rates determined by Hill functions with exponent two. Whilst a few transport processes occur by passive diffusion, in which case the flux is simply described by the concentration gradient, many transport processes are active and predominantly in one direction, so more complex descriptions are required. These are described on a case-by-case basis.

\subsection{Compartmentalisation}

Since our aim is to understand the biochemical complexity of the reaction and transport processes occurring in the human body, we minimise the structural information in our model. We propose a "lumped" model using a single concentration as representative of each chemical species in each tissue. We split the model into four compartments, that of liver, adipose tissue, skeletal muscle, and blood plasma, as the transport mechanism between the other three compartments. The roles of some other organs and tissues are discussed later in the derivation. 
The role of the liver in regulating the levels of metabolites available for other tissues means that any study of metabolism in the liver necessarily involves other tissues having a substantial turnover of these metabolites. Transport between the tissues is mediated through the blood plasma, since this involves all other tissues it is the most complicated to derive, and we leave the derivation of a governing equation for the blood plasma till last. The body's primary energy store adipose tissue, and skeletal muscle each have a substantial impact on metabolism, comprising approximately $20 \%$ and $40 \%$ respectively of the body by volume (in a healthy individual).

To account for the differences in the volume of compartments, we scale each differential equation by the size of the compartment which it concerns. The quantities $\alpha_{B}, \alpha_{L}, \alpha_{M}$, and $\alpha_{A}$ denote the volumes of blood, liver, skeletal muscle, and adipose tissue respectively. The full list of variables included in the model are summarised in Table 1. The overall scheme of reactions and transport is illustrated in Figure 1.

Note, throughout the model derivation, we assume a TAG molecule breaks down to form 3 FFAs, and similarly, 3 FFAs are required to form a TAG molecule.

\begin{tabular}{|rr||rr|}
\hline Liver & & Muscle \\
\hline Glucose & $G_{L}$ & Glucose & $G_{M}$ \\
Glycogen & $Y_{L}$ & Glycogen & $Y_{M}$ \\
G-6-P & $P_{L}$ & G-6-P & $P_{M}$ \\
Pyruvate & $R_{L}$ & Pyruvate & $R_{M}$ \\
FFA & $A_{L}$ & FFA & $A_{M}$ \\
TAG Storage Pool & $T_{L}$ & TAG & $T_{M}$ \\
TAG Secretory Pool & $S_{L}$ & Notional AMP & $P$ \\
\hline \hline Plasma & & Adipose & \\
\hline Glucose & $G_{B}$ & Glucose & $G_{A}$ \\
NEFA & $A_{N B}$ & FFA & $A_{A}$ \\
Endogenous TAG & $T_{L B}$ & TAG & $T_{A}$ \\
Exogenous TAG & $T_{C B}$ & Glycerol & $L_{A}$ \\
Insulin & $I$ & & \\
\hline
\end{tabular}

Table 1: Complete list of variables used in the model. 


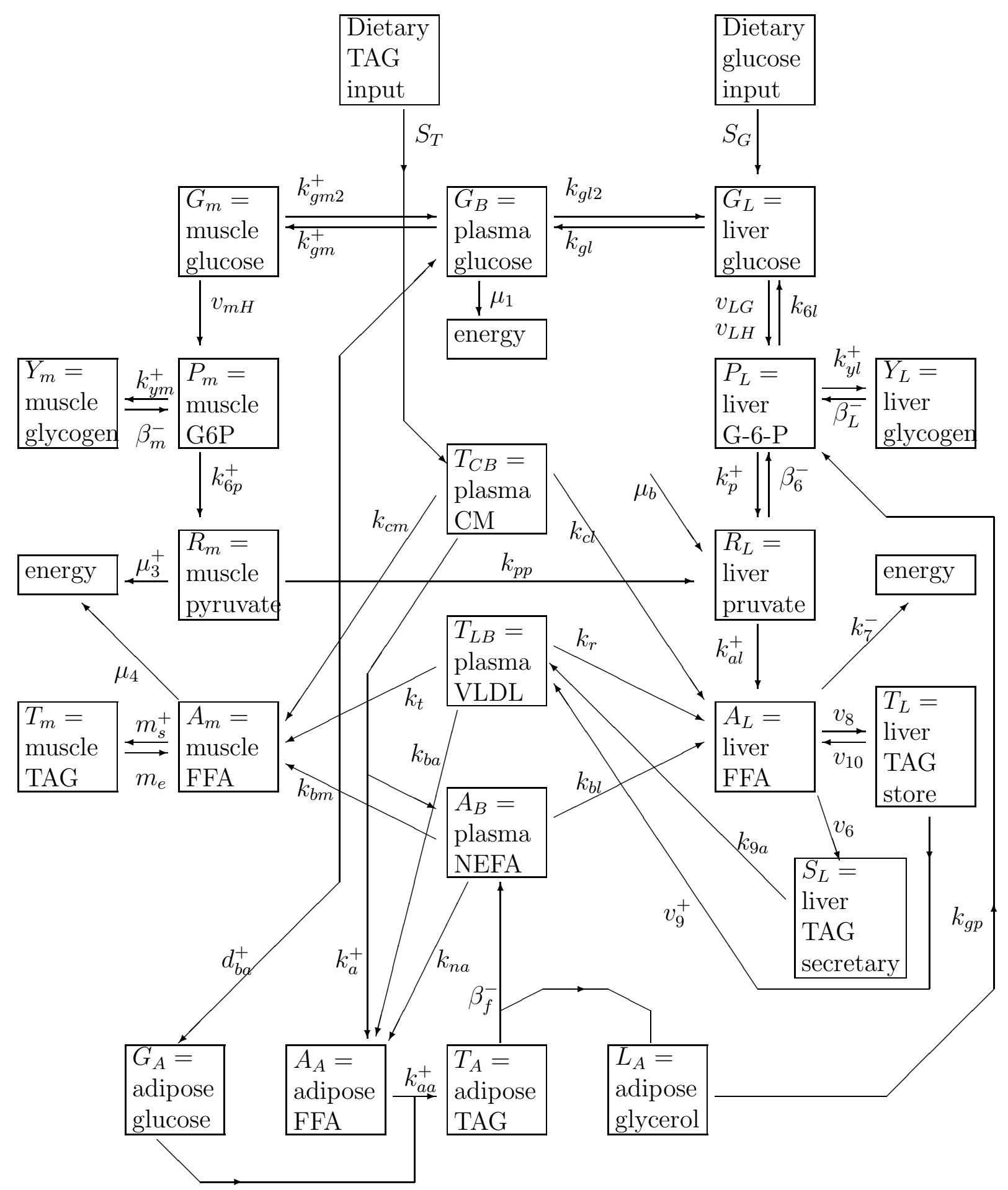

Figure 1: Model Diagram: at the top we show dietary input of glucose and TAG, on the right are liver components, on the left muscle, and at the bottom, adipose tissue. In the centre are plasma compartments. In addition to the variable names and descriptions, we include a parameter associated with each flux, plus and minus superscripts represent insulin stimulated and insulin inhibited pathways (respectively). 


\subsection{Dietary inputs}

Our model receives inputs from the gut from the dietary substrates TAG, glucose and fructose. We denote these by $S_{T}(t), S_{G}(t), S_{F}(t)$, respectively. The latter two are the only forms of carbohydrate we consider, the reason for separating these two is that the former elicits an insulin response whilst the latter does not. Sugars and fats enter the metabolic system in different ways. Glucose and fructose are both transported from the intestinal tract into the hepatic portal vein, from which they are eventually taken up by the liver. For simplicity, we choose not to explicitly include the glucose concentration in the hepatic portal vein, instead having a function adding dietary glucose directly to the liver glucose pool. Similarly for fructose, but once inside the liver it is converted (via several steps) to an intermediate in the glycolytic/gluconeogenic pathway. Fructose enters the pathway in the form of fructose-6-phosphate, an intermediate in the conversion of G-6-P to pyruvate. Since we aim to keep the number of variables to a minimum, we make the simplification that fructose enters the system as pyruvate in the liver. This simplification is valid, since fructose will almost certainly be ingested along side glucose, and therefore the glycolytic flux will be higher than that of gluconeogenic flux, forcing the metabolites towards pyruvate. Fatty acids differ in that they are packed into chylomicrons in the form of TAG and released through the lymphatic system before entering the blood stream. Hence, in our model, dietary fat enters via the plasma chylomicron TAG pool.

Analysis of glucose concentrations in plasma from experimental data [18] suggests the following functions are appropriate for our diet function

$$
S_{G}(t)=\alpha_{G} t e^{-t / \beta_{G}}, \quad S_{F}(t)=\alpha_{F} t e^{-t / \beta_{F}}, \quad S_{T}(t)=\alpha_{T} t e^{-t / \beta_{T}} .
$$

We assume similar shapes for the profiles of glucose, fructose and TAG, albeit with different parameters to reflect the different mechanisms by which these substrates are processed by the human body. Here the half-life of the absorption is approximately $\beta \ln 2$, and the total amount of each quantity taken up is $\alpha \beta^{2}$.

\subsection{Hormonal regulation}

Our model must be capable of simulating human metabolism in both fed and fasted states. The primary regulatory hormone involved in switching between the dominant 
active metabolic pathways in these two states is insulin. We ignore the effects of the counter-regulatory hormone glucagon for the moment as, in general, the two hormones have opposite effects; higher concentrations of insulin are seen when glucagon is low, and low concentrations when glucagon is high. In reality this relationship is more complicated, due to additional factors, such as glucagon stimulating the release of insulin, and some ketogenic and lipogenic pathways being affected by glucagon alone [37].

Glucose has the largest effect on the secretion of insulin, with amino acids, ketone bodies, and FFA having a significantly smaller effect, which we ignore in our model. Importantly, fructose does not elicit an insulin response, which is why we have treated it separately.

Experimental data of Harrison et al 22] suggests that insulin release (as a function of plasma glucose levels) takes the form of a sigmoidal function plus a basal secretion rate independent of glucose. Further experimental evidence [29] suggests the pancreatic beta cells have a Gaussian distribution of thresholds at which they release stored insulin. This relationship implies that the rate at which stored insulin is released is the integral of the Gaussian distribution, giving the error function [1] (in agreement with the sigmoidal shape predicted). Therefore we model the change in insulin by the sum of an error function and a basal secretion rate $\left(k_{11}\right)$, less a degradation term proportional to the amount of insulin, hence

$$
\underbrace{\frac{\mathrm{d} I}{\mathrm{~d} t}}_{\begin{array}{c}
\text { rate of change of } \\
\text { plasma insulin }
\end{array}}=\underbrace{k_{11}+k_{22} \operatorname{erf}\left(\frac{G_{B}-v}{c c}\right)}_{\begin{array}{c}
\text { Insulin production } \\
\text {-glucose stimuated }
\end{array}}-\underbrace{k_{d} I}_{\begin{array}{c}
\text { insulin } \\
\text { degradation }
\end{array}}
$$

where $v$ is the mean threshold value at which pancreatic beta cells release insulin.

\subsection{Liver}

\subsubsection{Liver carbohydrate}

Whilst the GLUT2 transporters present in the liver allow passive diffusion of glucose between plasma and liver, experimental data suggests this does not operate close to 
equilibrium (i.e Fick's laws may not apply). We assume that plasma glucose $\left(G_{B}\right)$ enters the liver at a rate proportional to plasma concentration with rate constant $k_{g l 2}$; similarly, glucose diffuses from the liver to the blood at a rate proportional to the liver concentration $\left(G_{L}\right)$ with rate constant $k_{g l}$.

To prevent a state of hyperglycemia, the liver traps large amounts of glucose in the form of glucose-6-phosphate (G-6-P). In the liver, this is handled by two enzymes, hexokinase and glucokinase. We assume Michaelis-Menten kinetic forms for these functions with rates $v_{L H}, v_{L G}$ and Michaelis-Menten constants $k_{L H}, k_{L G}$ respectively. There is added complexity in that hexokinase is strongly inhibited by its product, G-6-P $\left(P_{L}\right)$ via a noncompetitive inhibition with flux control coefficient $k_{r e p}$. The enzyme glucose-6-phosphatase reverses this process and is necessary for glucose release in fasted conditions. We assume this occurs at a rate proportional to the concentration of G-6-P, with rate constant $k_{6 l}$.

Including the diet function, $S_{G}(t)$, the equation for liver glucose is

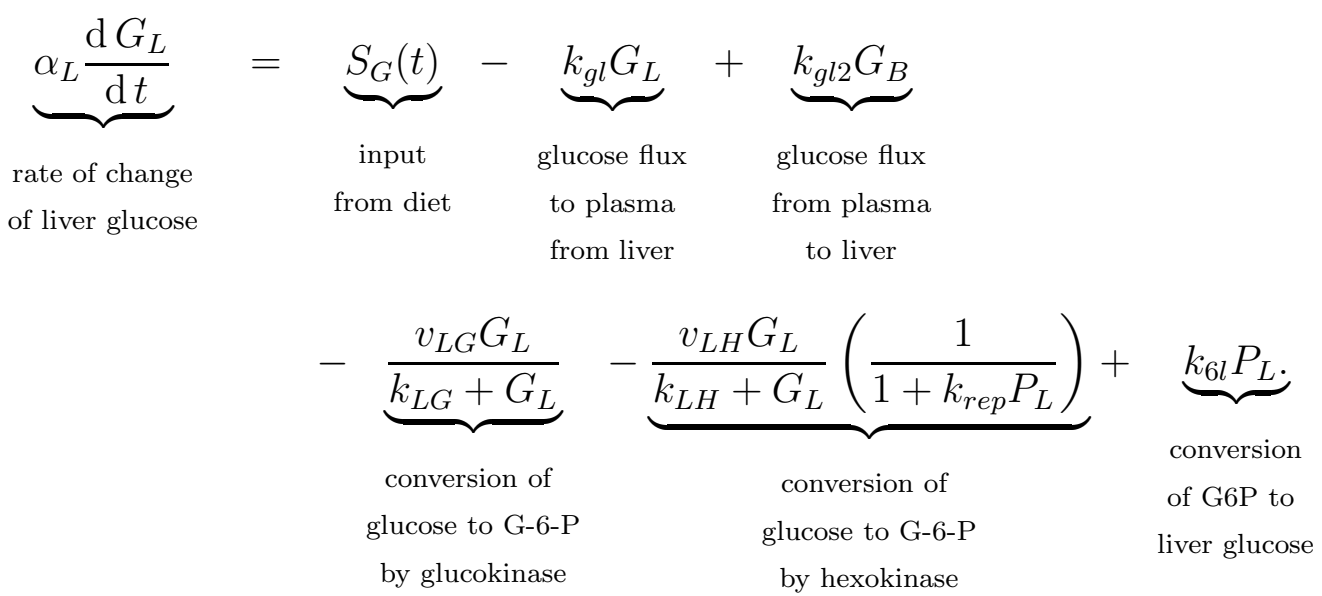

During high insulin conditions the liver stores glucose in the form of glycogen (glycogenesis) for future use, and breaks down glycogen to glucose (glycogenolysis) during periods of low insulin corresponding to low plasma glucose levels. As in the other mechanisms, glucose must first be converted to G-6-P, before being further metabolised to glycogen, we do not consider any intermediary steps. These glycogenesis/glycogenolysis pathways require a little more thought to model, as the liver has a finite glycogen storage capacity, which it will usually meet daily. 
We require glycogenesis to be roughly constant, until the liver is approaching its maximum storage capacity, at which time the uptake rates should reduce to zero. Defining the maximum glycogen store of the liver as $l_{\max }$ and introducing a small parameter $c_{0}$, we modify the glycogen synthesis by the smoothed step function $\frac{1}{2}(1+$ $\left.\tanh \left(\left(l_{\max }-Y_{L}\right) / c_{0}\right)\right)$, where $c_{0}$ represents the width of the range of glycogen values over which uptake drops to zero.

Similarly we require glycogenolysis to be roughly constant, until the liver has almost depleted its glycogen reserves at which point the release rate decreases continuously to zero. We therefore adjust the release term by the factor $Y_{L} /\left(Y_{L}+y_{0}\right)$, where $y_{0}$ is another small parameter, representing the range of $Y_{L}$ over which the release drops to zero as the glycogen store is depleted.

The actual rate of glycogen synthesis is stimulated by insulin, and is proportional to the concentration of the substrate available. We assign the rate $k_{y l}$ to this process. In our model the most immediate substrate is G-6-P. Degradation of glycogen is stimulated by glucagon, but since we consider only insulin in this model, we take the pathway to be insulin-inhibited, with rate constants $\beta_{L}, k_{d l}$. The equation to describe glycogen concentration in the liver is

$$
\underbrace{\alpha_{L} \frac{\mathrm{d} Y_{L}}{\mathrm{~d} t}}_{\begin{array}{c}
\text { of liver } \\
\text { glycogen }
\end{array}}=\underbrace{\frac{1}{2} k_{y l} I P_{L}\left(1+\tanh \left(\frac{l_{\text {max }}-Y_{L}}{c_{0}}\right)\right)}_{\begin{array}{c}
\text { conversion of G-6-P } \\
\text { to glycogen in the liver } \\
\text {-insulin-stimulated }
\end{array}}-\underbrace{\frac{\beta_{L}}{1+k_{d l} I}\left(\frac{Y_{L}}{Y_{L}+y_{0}}\right)}_{\begin{array}{c}
\text { release of liver glycogen } \\
\text { \& conversion to G-6-P }
\end{array}} .
$$

Glucose-6-phosphate is involved in many pathways, here it is also considered as the only intermediate between glucose and pyruvate in the glycolytic and gluconeogenic pathways. In general, the glycolytic pathway can be thought of as insulin-stimulated, and the gluconeogenic pathway insulin-inhibited. For the sake of simplicity, we assume the rate of conversion of G-6-P to pyruvate is proportional to the insulin concentration and the concentration of the substrate G-6-P, and depends on the rate parameter $k_{p}$. Conversely, conversion of pyruvate to G-6-P is proportional to the concentration of pyruvate, at an insulin inhibited rate, described by parameters $\beta_{6}$ and $k_{p 6}$. 
We also take into account the glycerol dynamics dictated by breakdown of triglyceride in adipose tissue (discussed in section 2.6). For now, we recognise that the liver takes up glycerol as a gluconeogenic precursor, and under fasting conditions it is metabolised within the liver to G-6-P. Intermediate glycerol concentrations in the plasma are ignored, thus uptake of G-6-P is modelled as being from adipose glycerol $\left(L_{A}\right)$, at rate $k_{g p}$. Therefore,

$$
\begin{aligned}
& \underbrace{\alpha_{L} \frac{\mathrm{d} P_{L}}{\mathrm{~d} t}}=-\underbrace{\frac{1}{2} k_{y l} I P_{L}\left(1+\tanh \left(\frac{l_{\max }-Y_{L}}{c_{0}}\right)\right)}+\underbrace{\frac{\beta_{L}}{1+k_{d l} I}\left(\frac{Y_{L}}{Y_{L}+y_{0}}\right)} \\
& \text { rate of change } \\
& \text { of G-6-P in } \\
& \text { the liver } \\
& +\underbrace{\frac{\beta_{6} R_{L}}{1+k_{p 6} I}}+\underbrace{\frac{v_{L G} G_{L}}{k_{L G}+G_{L}}}+\underbrace{\frac{v_{L H} G_{L}}{k_{L H}+G_{L}}\left(\frac{1}{1+k_{r e p} P_{L}}\right)} \\
& \begin{array}{ccc}
\text { gluconeogenesis } & \text { conversion of } & \text { conversion of } \\
\text { Pyr } \mapsto \text { G-6-P } & \text { glucose to G-6-P }
\end{array} \\
& \begin{array}{cr}
\text { in liver } & \text { by glucokinase }
\end{array} \\
& -\underbrace{k_{p} I P_{L}}-\underbrace{k_{6 l} P_{L}}+\underbrace{k_{g p} L_{A}} \\
& \text { conversion conversion adipose glycerol } \\
& \text { of G-6-P to of G-6-P to } \quad \mapsto \text { Liver G-6-P } \\
& \text { pyruvate liver glucose transport }
\end{aligned}
$$

Pyruvate kinetics in the liver $\left(R_{L}\right)$ are more complex. In addition to the glycolytic flux $\left(k_{6 l}\right)$ and gluconeogenic flux $\left(\beta_{6}, k_{p 6}\right)$ already described, we take into account de novo Lipogenesis, lactate uptake (from the Cori cycle), and glycerol return from hydrolysis of adipose triglyceride.

Under anaerobic conditions, and following a meal, glucose is the primary fuel oxidised in skeletal muscle, pyruvate cannot be fully oxidised, and lactate is produced. This is released by skeletal muscle and taken up by the liver where it is converted back to pyruvate. To keep the number of variables in the model to a minimum, we choose to ignore the concentrations of lactate in muscle, plasma, and liver, and instead consider muscle pyruvate to be directly transported to liver pyruvate (rate parameter 
$\left.k_{p p}\right)$. Additionally, red blood cells produce a constant amount of lactate, which is returned to the liver at rate $\mu_{b}$.

Whilst de novo lipogenesis (DNL) involves several reactions, the only metabolites in our model are pyruvate and the end product, FFA. We therefore choose our DNL term to convert liver pyruvate directly to FFA. DNL is stimulated by insulin, and is substrate-dependent, so initially we have DNL occurring at a rate $k_{a l}$ proportional to both insulin and pyruvate concentration $\left(R_{L}\right)$. The complete equation for pyruvate kinetics in the liver is

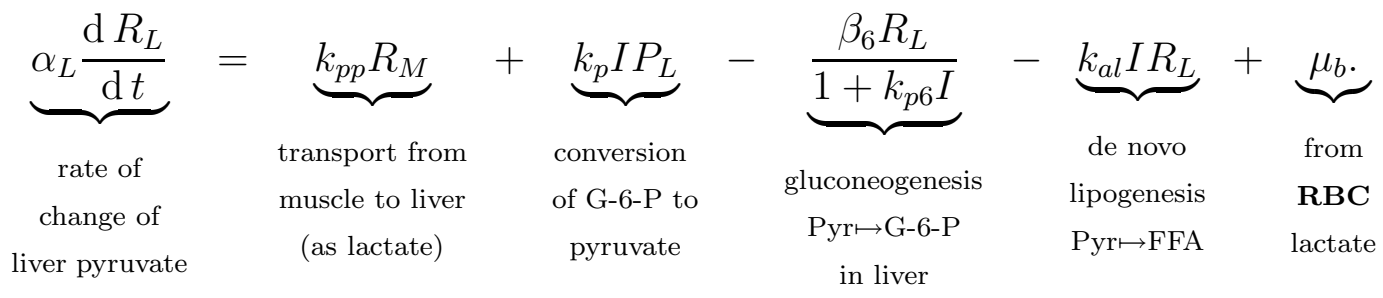

\subsubsection{Liver lipid}

We model fatty acid metabolism in the liver by considering three compartments, a TAG storage pool $\left(T_{L}\right)$, a TAG secretory pool $\left(S_{L}\right)$, and an intermediate FFA pool $\left(A_{L}\right)$. Plasma NEFA, lipoprotein remnants and hepatic DNL contribute to the intermediate FFA pool as previously described. The number of particles secreted, and therefore the amount of FFA entering the immediate secretory pathway is modelled as a saturating function of the concentration $A_{L}$, with rate parameters $v_{6}, k_{6}$. Alternatively, intermediate FFA can be stored in the cytosol of hepatocytes. We assume this process also saturates, with associated parameters, $v_{8}, k_{8}$. Beta-oxidation of fatty acids has been shown to decrease in response to insulin [60], so we assume a loss of FFA proportional to the amount of FFA at rate $k_{7}$, and at an insulin-inhibited rate $k_{5}$. DNL adds to liver fatty acids at rate $k_{a l}$ as previously described. Thus, the equation for hepatic FFA is

$$
\underbrace{\alpha_{L} \frac{\mathrm{d} A_{L}}{\mathrm{~d} t}}_{\begin{array}{c}
\text { rate of } \\
\text { change of }
\end{array}}=\underbrace{3 k_{c l} T_{C B}}_{\begin{array}{c}
\text { uptake of } \\
\text { exogenous plasma } \\
\text { TAG in liver }
\end{array}}+\underbrace{k_{b l} A_{N B}}_{\begin{array}{c}
\text { FFA transport } \\
\text { from plasma } \\
\text { to liver }
\end{array}}+\underbrace{3 k_{r} T_{L B}}_{\begin{array}{c}
\text { from } \\
\text { plasma TAG } \\
\text { (endogenous) }
\end{array}}+\underbrace{k_{a l} I R_{L}}_{\begin{array}{c}
\text { de novo } \\
\text { lipogenesis } \\
\text { Pyr } \mapsto F F A
\end{array}}
$$




$$
\begin{aligned}
& +\underbrace{\frac{3 v_{10} T_{L}}{k_{10}+T_{L}}}-\underbrace{\frac{3 v_{6} A_{L}}{k_{6}+A_{L}}}-\underbrace{\frac{3 v_{8} A_{L}}{k_{8}+A_{L}}}-\underbrace{\frac{k_{7} A_{L}}{1+k_{5} I}} . \\
& \begin{array}{cccc}
\text { TAG storage } & \text { Liver FFA } & \text { FFA } & \beta \text {-oxidation } \\
\text { conversion } & \text { input to TAG } & \text { conversion to } & \text { of FFA }
\end{array} \\
& \text { to FFA secretary pool TAG storage }
\end{aligned}
$$

TAG transferred to the secretory pool is released into plasma lipoproteins at a rate proportional to the amount stored, therefore

$$
\underbrace{\alpha_{L} \frac{\mathrm{d} S_{L}}{\mathrm{~d} t}}_{\begin{array}{c}
\text { rate of change } \\
\text { of TAG secretary } \\
\text { pool in liver }
\end{array}}=\underbrace{\frac{v_{6} A_{L}}{k_{6}+A_{L}}}_{\begin{array}{c}
\text { Liver FFA } \\
\text { input to TAG } \\
\text { secretary pool }
\end{array}}-\underbrace{\text { pool to plas }}_{\begin{array}{r}
\text { poop to plasma } \\
\text { from secretary }
\end{array}}
$$

The bulk addition of cytosolic stored TAG depends on the amount of TAG stored and on hormonal regulation. Experimental evidence of Adiels et al [3] suggests that the release of TAG is a saturating function of liver fat with rate parameters $v_{9}, k_{9}$.

Insulin's effect on VLDL metabolism is to down-regulate the quantity of VLDL1 secreted, and up-regulate the quantity of VLDL2 secreted [4]. We let the term $F(I)$ denote the fraction of VLDL2 which is further lipidated to form the larger VLDL1 particles. Thus the equation for hepatic cytosolic TAG is

$$
\underbrace{\alpha_{L} \frac{\mathrm{d} T_{L}}{\mathrm{~d} t}}_{\substack{\text { TAG storage } \\
\text { pool in liver }}}=\underbrace{\frac{v_{8} A_{L}}{k_{8}+A_{L}}}_{\begin{array}{c}
\text { FFA } \\
\text { conversion } \\
\text { to TAG }
\end{array}}-\underbrace{F(I) \frac{v_{9} T_{L}}{k_{9}+T_{L}}}_{\begin{array}{c}
\text { release } \\
\text { of TAG into } \\
\text { plasma }
\end{array}}-\underbrace{\frac{v_{10} T_{L}}{k_{10}+T_{L}}}_{\begin{array}{c}
\text { TAG } \\
\text { conversion } \\
\text { to FFA }
\end{array}} .
$$

The choice of $F(I)$ is detailed in the parameter estimation section, we assume $F(I)$ takes the form

$$
F(I)=k_{12} \tanh \left(\frac{v_{12}-I}{k_{13}}\right)+k_{14} .
$$

Note that since $k_{12}<k_{14}$ (see data in Table 3), this effect is not large, as shown by the data of Søndergaard et al. [52]. 


\subsection{Skeletal muscle}

\subsubsection{Skeletal muscle carbohydrate}

The storage and use of glucose in skeletal muscle is based on the same mechanisms as in the liver, but with some fundamental differences, and different values for equivalent parameters. The concentration of glucose-6-phosphatase (the enzyme used in catalysing G-6-P to glucose) in skeletal muscle is incredibly low, and hence, we ignore this reverse pathway. Another enzyme found in low concentrations in skeletal muscle is pyruvate carboxylase, the first enzyme needed for gluconeogenesis from the precursor pyruvate. Hence, we ignore the conversion of pyruvate to G-6-P.

Diffusion of glucose between plasma and tissue also differs, with the addition of GLUT4 transporters present in skeletal muscle. We assume the number of GLUT4 transporters increases in proportion to the concentration of insulin. Thus the permeability of the surface can be thought of as the combination of GLUT1 transporters (represented by the rate $k_{g}$ ) and of the GLUT4 transporters (represented by the rate $\left.k_{g i} I\right)$. Again, experimental evidence suggests that this diffusion acts far from equilibrium, so exchange from blood to muscle occurs at rate different from that of muscle to blood at rate $k_{g m 2}$.

Unlike the liver, skeletal muscle contains no glucokinase, so phosphorylation is constrained to hexokinase only. We assume the same form for muscle hexokinase activity (and G-6-P inhibition) as in the liver, here the associated constants are $v_{M H}, k_{M H}$, and $k_{r e p}$. Thus the equation describing the evolution of glucose in skeletal muscle is

$$
\underbrace{\alpha_{M} \frac{\mathrm{d} G_{M}}{\mathrm{~d} t}}_{\begin{array}{c}
\text { rate of change } \\
\text { of glucose } \\
\text { in muscle }
\end{array}}=\underbrace{\left(1+k_{g i} I\right)\left(k_{g m} G_{B}-k_{g m 2} G_{M}\right)}_{\begin{array}{c}
\text { glucose transport } \\
\text { between plasma and muscle } \\
\text {-insulin stimulated }
\end{array}}-\underbrace{\frac{v_{M H} G_{M}}{k_{M H}+G_{M}}\left(\frac{1}{1+k_{r e p} P_{M}}\right)}_{\begin{array}{c}
\text { conversion of glucose to G-6-P } \\
\text { by hexokinase } \\
\text {-inhibited by G-6-P }
\end{array}} .
$$

Glycogen storage and release in skeletal muscle operates in the same way as in the liver, albeit with different parameters; hence we define the glycogen synthesis rate by $k_{y m}$, and have degradation parameters $\beta_{m}, k_{d y} I$, with a maximum glycogen concen- 
tration of $m_{\max }$. The equation for muscle glycogen is thus

$$
\begin{aligned}
& \underbrace{\alpha_{M} \frac{\mathrm{d} Y_{M}}{\mathrm{~d} t}}=\underbrace{\frac{1}{2} k_{y m} I P_{M}\left(1+\tanh \left(\frac{m_{\max }-Y_{M}}{c_{0}}\right)\right)}-\underbrace{\frac{\beta_{m}}{1+k_{d y} I}\left(\frac{Y_{M}}{Y_{M}+y_{0}}\right)} . \\
& \text { rate of } \\
& \text { change of } \\
& \text { muscle glycogen } \\
& \text { in muscle }
\end{aligned}
$$

The glucose-6-phosphate concentration in skeletal muscle follows from the fluxes and assumptions detailed above; however, the glycolytic flux out of G-6-P in skeletal muscle occurs at a different rate to the liver, here the rate is taken to be $k_{6 p}$, hence

$$
\begin{aligned}
& \underbrace{\alpha_{M} \frac{\mathrm{d} P_{M}}{\mathrm{~d} t}}=\underbrace{\frac{v_{M H} G_{M}}{k_{M H}+G_{M}}\left(\frac{1}{1+k_{r e p} P_{M}}\right)}-\underbrace{\frac{1}{2} k_{y m} I P_{M} \cdot\left(1+\tanh \left(\frac{m_{\max }-Y_{M}}{c_{0}}\right)\right)} \\
& \text { rate of } \\
& \text { change of } \\
& \text { muscle G-6-P }
\end{aligned}
$$

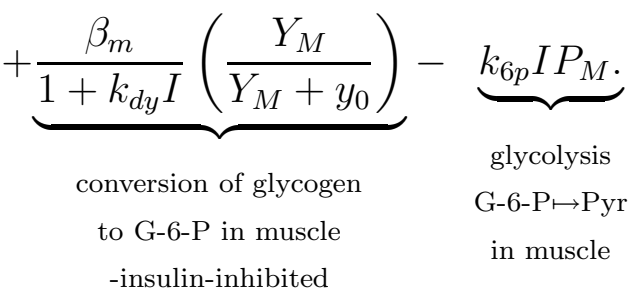

The pyruvate concentration in skeletal muscle is dictated by three components: substrate entering through the glycolytic pathway $\left(k_{6 p}\right)$, pyruvate release for uptake by the liver (as lactate with rate parameter $k_{p p}$ ), and consumption by skeletal muscle. Carbohydrate oxidation in skeletal muscle is covered in detail in section 2.5.3, here we have modelled it by the term $\mu_{3} R_{M} I P$, thus the equation for muscle pyruvate is

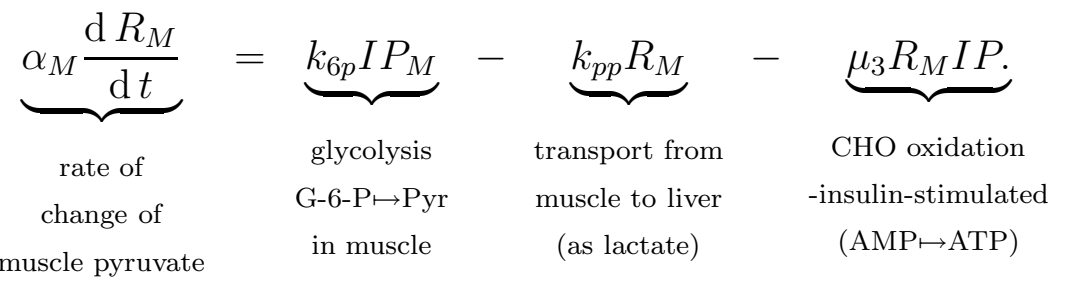

\subsubsection{Skeletal muscle lipid}

For fat metabolism in skeletal muscle, we are concerned only with a TAG storage pool and a FFA pool. The FFA pool is contributed to by chylomicron TAG, LP TAG and 
NEFA, with fluxes proportional to concentration at rates $k_{c m}, k_{t}$ and $k_{b m}$ respectively. Skeletal muscle stores FFA in the form of TAG at the insulin-dependent rate $m_{s}$, and releases TAG for energy when required. For simplicity, we assume that the breakdown of TAG occurs at a constant rate, $m_{e}$, thereby always ensuring an adequate energy supply. FFA oxidation is dictated by the term $\mu_{3} R_{M} I P$, as discussed in section 2.5.3. The equations for FFA and TAG in skeletal muscle are

$$
\begin{aligned}
& \underbrace{\alpha_{M} \frac{\mathrm{d} A_{M}}{\mathrm{~d} t}}_{\begin{array}{c}
\text { rate of } \\
\text { change of }
\end{array}}=-\underbrace{3 m_{s} I A_{M}}_{\begin{array}{c}
\text { FFA } \mapsto \text { TAG } \\
\text { in muscle }
\end{array}}+\underbrace{3 m_{e}}_{\begin{array}{c}
\text { TAG } \mapsto \mathrm{FFA} \\
\text { in muscle }
\end{array}}+\underbrace{3 k_{c m} T_{C B}}_{\begin{array}{c}
\text { uptake of plasma } \\
\text { TAG (exogenous) }
\end{array}}+\underbrace{\text { into muscle }_{b m} A_{N B}}_{\begin{array}{c}
\text { FFA uptake } \\
\text { from plasma } \\
\text { into muscle FFA }
\end{array}} \\
& +\underbrace{3 k_{t} T_{L B}}_{\begin{array}{c}
\text { uptake of plasma } \\
\text { TAG (endogenous) }
\end{array}}-\underbrace{\mu_{4} A_{M} P}_{\begin{array}{c}
\text { FFA } \\
\text { oxidation }
\end{array}} \\
& \text { into muscle FFA } \quad(\mathrm{AMP} \mapsto \mathrm{ATP})
\end{aligned}
$$

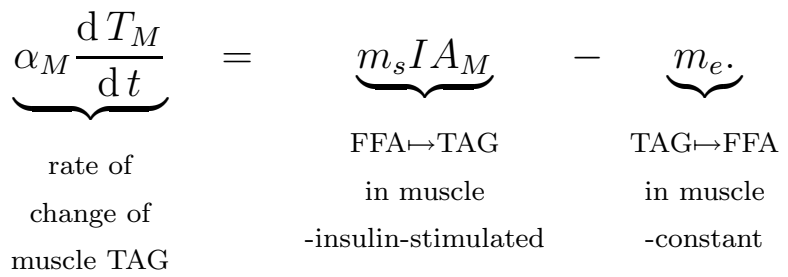

\subsubsection{Skeletal muscle substrate oxidation}

Skeletal muscle oxidation patterns demonstrate an ability to switch fuel choice from mostly fatty acids in the fasted state, to predominantly glucose in the fed state, facilitating the disposal of a carbohydrate load. We introduce the equation describing $P$, a notional AMP concentration. Energy expenditure is assumed to be constant, thus AMP is created at a constant rate $\mu_{a m p}$. AMP is converted back to ATP via fuel oxidation. Fatty acid oxidation contributes to the replenishment of AMP to ATP at a rate proportional to the concentration of available free fatty acids, $A_{M}$ and to the amount of AMP that needs replenishing $(P)$ with the constant of proportionality being $\mu_{4}$. In a similar way, carbohydrates also contribute to reducing $P$, but with the additional influence of insulin in promoting the use of carbohydrate as a fuel source. For simplicity we assume insulin exerts this effect linearly. Again, oxidation is proportional to the amount of AMP that needs replenishing $(P)$ and to the availability of substrates, in this case, pyruvate $\left(R_{M}\right)$, as the last metabolite modelled in the 
glycolytic pathway. Hence $P$ is governed by

$$
\underbrace{\frac{\mathrm{d} P}{\mathrm{~d} t}}_{\begin{array}{c}
\text { rate of } \\
\text { change of }
\end{array}}=\underbrace{\mu_{\text {amp }}}_{\begin{array}{c}
\text { Production of } \\
\text { AMP from ATP } \\
\text { by exercise }
\end{array}}-\underbrace{\mu_{4} A_{M} P}_{\begin{array}{c}
\text { FFA } \\
\text { oxidation } \\
\text { AMP } \mapsto \text { ATP }
\end{array}}-\underbrace{\mu_{3} R_{M} I P}_{\begin{array}{c}
\text { CHO oxidation } \\
\text {-insulin-stimulated } \\
\text { AMP } \mapsto \text { ATP }
\end{array}}
$$

\subsection{Adipose tissue}

Whilst we only model FFA and TAG in the lipid storage dynamics of skeletal muscle and the liver, adipose tissue requires the consideration of additional species. TAG is formed from a combination of a glycerol-3-phosphate backbone (a product of glycolysis) and three fatty acids. When TAG is hydrolysed by lipases in adipose tissue, the backbone is released in the form of glycerol alone, i.e no phosphate group is attached. Since adipose tissue lacks the enzyme required to convert glycerol to G-3-P (glycerol kinase), glycerol leaves the cell, and a new G-3-P must be synthesised. Two G-3-P molecules can be synthesised from one glucose molecule. The glycerol leaving adipose tissues is returned to the liver where it can be used as a gluconeogenic precursor. We do not model the concentration of G-3-P, instead we consider these processes to involve only glucose, FFA, TAG and glycerol.

TAG is synthesised from available free fatty acids $\left(A_{A}\right)$, and a glycerol backbone (synthesised from glucose $G_{A}$ ). This process is stimulated by insulin at rate $k_{a a}$. Adipose TAG is broken down to FFA for release as NEFA in fasted conditions. The main enzyme responsible for this (hormone sensitive lipase) is inhibited by insulin. Note, in parameterising this process to data, we required inhibition to be proportional to insulin squared. Importantly, since the concentration of adipose TAG in a healthy person would be well above any substrate saturating level, we model the breakdown and release of TAG with no substrate dependence. Thus TAG breakdown to FFA and immediate release into NEFA is modelled by the rate $\beta_{f}$, and inhibited by insulin with parameter $k_{f t}$. The equation for adipose TAG is thus

$$
\underbrace{\alpha_{A} \frac{\mathrm{d} T_{A}}{\mathrm{~d} t}}_{\begin{array}{c}
\text { rate of } \\
\text { change of } \\
\text { adipose TAG }
\end{array}}=\underbrace{k_{a a} I A_{A} G_{A}}_{\begin{array}{c}
\text { TAG production } \\
\text { from FFA and } \\
\text { glucose/glycerol }
\end{array}}-\underbrace{\frac{\beta_{f}}{1+k_{f t} I^{2}}}_{\begin{array}{c}
\text { Release of adipose } \\
\text { TAG to plasma FFA } \\
\text {-insulin-inhibited }
\end{array}}
$$


Adipose tissue takes up TAG from chylomicrons, and from lipoproteins released from the liver. LPL, which is responsible for removing this TAG from the lipoproteins, acts at a basal rate in fasted conditions, but its activity increases in response to insulin. Note, LPL activity is only up-regulated by insulin in adipose tissue, it is unchanged or decreased in skeletal muscle [17]. We therefore model TAG uptake as proportional to the concentrations of the respective lipoproteins, and the sum of basal $\left(k_{a}, k_{b a}\right)$ and insulin-stimulated rates $\left(k_{a i}\right)$. Note, the action of LPL on the lipoproteins breaks down this TAG to FFA as it crosses the tissue lining, thus lipoprotein derived TAG adds to the adipose FFA pool $\left(A_{A}\right)$, not the TAG pool. This process is inherently 'leaky' and thus a proportion $k_{l p}$ is transferred to the plasma. The conversion of FFA to TAG is modelled as proportional to concentrations of fatty acids, glucose, and to insulin as previously described (with rate parameter $k_{a a}$ ). Additionally, adipose can take up NEFA from the pool released by adipose TAG. This takes place at rate $k_{n a}$ proportional to the NEFA concentration. Thus the equation for adipose FFA is

$$
\begin{gathered}
\underbrace{\alpha_{A} \frac{\mathrm{d} A_{A}}{\mathrm{~d} t}}_{\begin{array}{c}
\text { rate of } \\
\text { change of } \\
\text { adpipose FFA }
\end{array}}=-\underbrace{3 k_{a a} I A_{A} G_{A}}_{\begin{array}{c}
\text { TAG production } \\
\text { from FFA and } \\
\text { glucose/glycerol }
\end{array}}+\underbrace{3 k_{a}\left(1+k_{a i} I\right)\left(1-k_{l p}\right) T_{C B}}_{\begin{array}{c}
\text { uptake of plasma } \\
\text { TAG (exogenous) } \\
\text { by adipose tissue }
\end{array}} \\
+\underbrace{3 k_{b a} T_{L B}}_{\begin{array}{c}
\text { uptake of plasma } \\
\text { TAG (endogenous) } \\
\text { into Adipose FFA }
\end{array}}+\underbrace{k_{n a} A_{N B}}_{\begin{array}{c}
\text { uptake of } \\
\text { plasma FFA } \\
\text { into adipose FFA }
\end{array}}
\end{gathered}
$$

The glycerol backbone produced in the breakdown of TAG is released at the same rate as adipose TAG is broken down, the associated rate parameters being $\beta_{f}, k_{f t}$. The glycerol released into the plasma is taken up by the liver. We do not explicitly model the concentration of glycerol in the plasma, instead, we model the direct uptake of adipose glycerol to the liver at a rate $k_{g p}$ proportional to the adipose glycerol concentration $L_{A}$, hence

$$
\underbrace{\alpha_{A} \frac{\mathrm{d} L_{A}}{\mathrm{~d} t}}_{\begin{array}{c}
\text { rate of } \\
\text { change of } \\
\text { adipose glycerol }
\end{array}}=\underbrace{\frac{\beta_{f}}{1+k_{f t} I^{2}}}_{\begin{array}{c}
\text { Release of adipose TAG } \\
\text { to plasma FFA-insulin-inhibited } \\
\text { also releasing glycerol }
\end{array}}-\underbrace{k_{g p} L_{A}}_{\begin{array}{c}
\text { adipose glycerol } \\
\text { Liver G-6-P } \\
\text { transport }
\end{array}}
$$


The major supply of G-3-P necessary for the storage of FFA as TAG is sourced from plasma glucose and metabolised to G-3-P within adipose tissue. Rather than model every metabolite in the glycolytic pathway leading to G-3-P, we consider adipose glucose as a proxy for G-3-P. Taking into account GLUT1 $\left(d_{B A}\right)$, and GLUT4 $\left(k_{g a}\right)$ transporters (in a similar manner as to skeletal muscle), we allow for the diffusion of glucose between plasma $\left(G_{B}\right)$ and adipose glucose $\left(G_{A}\right)$. As previously described, this glucose is metabolised to G-3-P for esterification with FFA at a rate $k_{a a}$, hence

$$
\underbrace{\alpha_{A} \frac{\mathrm{d} G_{A}}{\mathrm{~d} t}}_{\begin{array}{c}
\text { rate of change } \\
\text { of glucose in } \\
\text { adipose tissue }
\end{array}}=\underbrace{d_{B A}\left(1+k_{g a} I\right)\left(G_{B}-G_{A}\right)}_{\begin{array}{c}
\text { glucose transport } \\
\text { between plasma } \\
\text { and adipose tissue }
\end{array}}-\underbrace{k_{a a} I A_{A} G_{A}}_{\begin{array}{c}
\text { TAG production } \\
\text { from FFA and } \\
\text { glucose/glycerol }
\end{array}} .
$$

\subsection{Plasma variables}

The evolution of the plasma variables consist of fluxes between compartments already described, as well as usage by other tissues. A short summary of terms is given below.

As previously discussed, dietary fat $S_{F}(t)$ adds to the chylomicron TAG pool, and is taken up by skeletal muscle $\left(k_{c m}\right)$, adipose tissue $\left(k_{a}, k_{a i}\right)$ and liver $\left(k_{c l}\right)$, thus the concentration $T_{C B}$ is given by

$$
\begin{aligned}
& \underbrace{\frac{\mathrm{d} T_{C B}}{\mathrm{~d} t}}=\underbrace{S_{F}(t)}-\underbrace{k_{c m} T_{C B}}-\underbrace{k_{c l} T_{C B}}-\underbrace{k_{a}\left(1+k_{a i} I\right) T_{C B}} . \\
& \text { rate of change input uptake of plasma uptake of uptake of plasma } \\
& \text { of exogenous from TAG (exogenous) exogenous plasma TAG TAG (exogenous) } \\
& \text { TAG in plasma diet into muscle FFA into liver FFA by adipose tissue }
\end{aligned}
$$

NEFA is released by adipose tissue $\left(\beta_{a}, k_{a b}\right)$, and taken up by the liver $\left(k_{b l}\right)$, adipose tissue $\left(k_{n a}\right)$, and by muscle $\left(k_{b m}\right)$. Additionally, the action of LPL on circulating lipoproteins leaks fatty acids into the plasma at a rate $k_{l p}$ proportional to the TAG extracted.

$$
\underbrace{\frac{\mathrm{d} A_{N B}}{\mathrm{~d} t}}_{\begin{array}{c}
\text { rate of } \\
\text { change of } \\
\text { plasma FFA }
\end{array}}=-\underbrace{k_{b m} A_{N B}}_{\begin{array}{c}
\text { FFA uptake } \\
\text { from plasma } \\
\text { into muscle }
\end{array}}-\underbrace{k_{b l} A_{N B}}_{\begin{array}{c}
\text { FFA transport } \\
\text { from plasma } \\
\text { to liver }
\end{array}}-\underbrace{k_{\text {into adipose FFA }}}_{\begin{array}{c}
\text { uptake of } \\
\text { plasma FFA }
\end{array}}
$$




$$
+\underbrace{\frac{3 \beta_{f}}{1+k_{f t} I^{2}}}_{\begin{array}{c}
\text { Release of adipose TAG } \\
\text { to plasma FFA-insulin-inhibited } \\
\text { also releasing glycerol }
\end{array}}+\underbrace{3 k_{a} k_{l p}\left(1+k_{a i} I\right) T_{C B}}_{\begin{array}{c}
\text { adipose tissue uptake } \\
\text { of TAG from plasma } \\
\text { (exogenous) }
\end{array}} .
$$

Endogenously derived lipoprotein TAG $\left(T_{L B}\right)$ is secreted from the liver $\left(v_{9}, k_{9}, k_{9 a}\right)$ and taken up by skeletal muscle $\left(k_{t}\right)$, adipose tissue $\left(k_{b a}\right)$, and as remnant particles by the liver $\left(k_{r}\right)$, hence

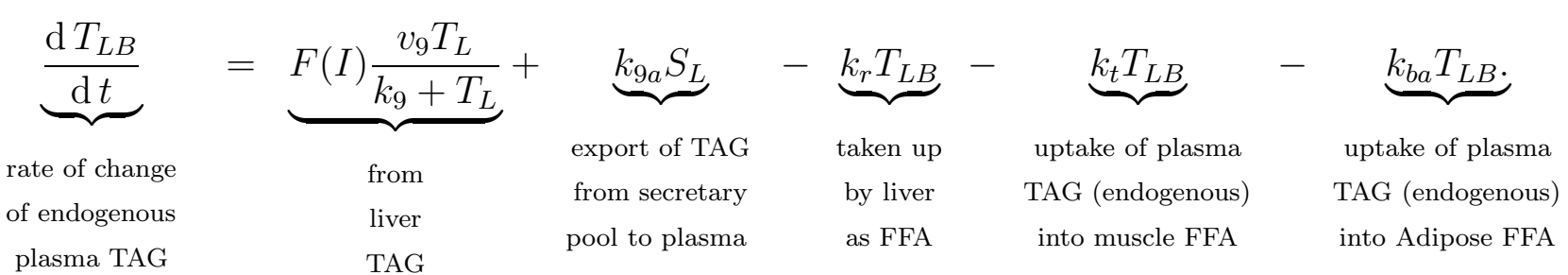

Ebbert and Jensen [16] review the literature on free fatty acid metabolism and the effects of high levels. They note that upto $50 \%$ of NEFA comes from visceral fat, but do not comment on the effect of subcutaneous fats. Whilst the work of Jensen et al. [28] provides a more detailed analysis of FFA kinetics, their data is from dogs, not humans.

Plasma glucose diffuses between liver $\left(k_{g l}, k_{g l 2}\right)$, skeletal muscle $\left(k_{g}, k_{g i}, k_{g m}, k_{g m 2}\right)$ and adipose $\left(k_{g a}, d_{B A}\right)$ as previously described. Plasma glucose can be used as a fuel source by a number of organs/cells, the largest two consumers being the brain and red blood cells. Since these consumers use glucose at an almost constant rate, we introduce a term for the removal of glucose at constant rate $\mu_{1}$

$$
\begin{aligned}
& \underbrace{\frac{\mathrm{d} G_{B}}{\mathrm{~d} t}}_{\text {rate of }}=\underbrace{k_{g l} G_{L}}_{\text {glucose flux }}-\underbrace{k_{g l 2} G_{B}}_{\text {glucose flux }}-\underbrace{\left(1+k_{g i} I\right)\left(k_{g m} G_{B}-k_{g m 2} G_{M}\right)}_{\text {glucose transport }} \\
& \text { change of to plasma from plasma between plasma and muscle } \\
& \text { plasma glucose from liver to liver -insulin-stimulated } \\
& -\underbrace{-d_{B A}\left(1+k_{g a} I\right)\left(G_{B}-G_{A}\right)}_{\begin{array}{c}
\text { glucose transport } \\
\text { between plasma } \\
\text { and adipose tissue }
\end{array}}-\underbrace{\mu_{1}}_{\begin{array}{c}
\text { use of } \\
\text { glucose } \\
\text { as fuel }
\end{array}}
\end{aligned}
$$

This completes the model. 


\subsection{Summary}

The variables and transport processes are summarised in Figure1, We now summarise the equations derived above.

$$
\begin{aligned}
& \frac{\mathrm{d} I}{\mathrm{~d} t}=k_{11}+k_{22} \operatorname{erf}\left(\frac{G_{B}-v}{c c}\right)-k_{d} I \\
& \alpha_{L} \frac{\mathrm{d} G_{L}}{\mathrm{~d} t}=S_{G}(t)-k_{g l} G_{L}+k_{g l 2} G_{B}-\frac{v_{L G} G_{L}}{k_{L G}+G_{L}}-\frac{v_{L H} G_{L}}{k_{L H}+G_{L}}\left(\frac{1}{1+k_{r e p} P_{L}}\right)+k_{6 l} P_{L} \\
& \alpha_{L} \frac{\mathrm{d} Y_{L}}{\mathrm{~d} t}=\frac{1}{2} k_{y l} I P_{L}\left(1+\tanh \left(\frac{l_{\max }-Y_{L}}{c_{0}}\right)\right)-\frac{\beta_{L}}{1+k_{d l} I}\left(\frac{Y_{L}}{Y_{L}+y_{0}}\right), \\
& \alpha_{L} \frac{\mathrm{d} P_{L}}{\mathrm{~d} t}=-\frac{1}{2} k_{y l} I P_{L}\left(1+\tanh \left(\frac{l_{\max }-Y_{L}}{c_{0}}\right)\right)+\frac{\beta_{L}}{1+k_{d l} I}\left(\frac{Y_{L}}{Y_{L}+y_{0}}\right)-k_{p} I P_{L}+k_{g p} L_{A} \\
& +\frac{\beta_{6} R_{L}}{1+k_{p 6} I}+\frac{v_{L G} G_{L}}{k_{L G}+G_{L}}+\frac{v_{L H} G_{L}}{k_{L H}+G_{L}}\left(\frac{1}{1+k_{r e p} P_{L}}\right)-k_{6 l} P_{L} \\
& \alpha_{L} \frac{\mathrm{d} R_{L}}{\mathrm{~d} t}=k_{p p} R_{M}+k_{p} I P_{L}-\frac{\beta_{6}}{1+k_{p 6} I} R_{L}-k_{a l} I R_{L}+\mu_{b}, \\
& \alpha_{L} \frac{\mathrm{d} A_{L}}{\mathrm{~d} t}=3 k_{c l} T_{C B}+k_{b l} A_{N B}+3 k_{r} T_{L B}+k_{a l} I R_{L}-\frac{3 v_{6} A_{L}}{k_{6}+A_{L}} \\
& +\frac{3 v_{10} T_{L}}{k_{10}+T_{L}}-\frac{3 v_{8} A_{L}}{k_{8}+A_{L}}-\frac{k_{7} A_{L}}{1+k_{5} I} \\
& \alpha_{L} \frac{\mathrm{d} S_{L}}{\mathrm{~d} t}=\frac{v_{6} A_{L}}{k_{6}+A_{L}}-k_{9 a} S_{L} \\
& \alpha_{L} \frac{\mathrm{d} T_{L}}{\mathrm{~d} t}=\frac{v_{8} A_{L}}{k_{8}+A_{L}}-F(I) \frac{v_{9} T_{L}}{k_{9}+T_{L}}-\frac{v_{10} T_{L}}{k_{10}+T_{L}}, \\
& \alpha_{M} \frac{\mathrm{d} G_{M}}{\mathrm{~d} t}=\left(1+k_{g i} I\right)\left(k_{g m} G_{B}-k_{g m 2} G_{M}\right)-\frac{v_{M H} G_{M}}{k_{M H}+G_{M}}\left(\frac{1}{1+k_{r e p} P_{M}}\right) \text {, } \\
& \alpha_{M} \frac{\mathrm{d} Y_{M}}{\mathrm{~d} t}=\frac{1}{2} k_{y m} I P_{M}\left(1+\tanh \left(\frac{m_{\max }-Y_{M}}{c_{0}}\right)\right)-\frac{\beta_{m}}{1+k_{d y} I}\left(\frac{Y_{M}}{Y_{M}+y_{0}}\right), \\
& \alpha_{M} \frac{\mathrm{d} P_{M}}{\mathrm{~d} t}=\frac{v_{M H} G_{M}}{k_{M H}+G_{M}}\left(\frac{1}{1+k_{r e p} P_{M}}\right)-k_{y m} I P_{M} \cdot \frac{1}{2}\left(1+\tanh \left(\frac{m_{\max }-Y_{M}}{c_{0}}\right)\right) \\
& +\frac{\beta_{m}}{1+k_{d y} I}\left(\frac{Y_{M}}{Y_{M}+y_{0}}\right)-k_{6 p} I P_{M}, \\
& \alpha_{M} \frac{\mathrm{d} R_{M}}{\mathrm{~d} t}=k_{6 p} I P_{M}-k_{p p} R_{M}-\mu_{3} R_{M} I P, \\
& \alpha_{M} \frac{\mathrm{d} A_{M}}{\mathrm{~d} t}=-3 m_{s} I A_{M}+3 m_{e}+3 k_{c m} T_{C B}+k_{b m} A_{N B}+3 k_{t} T_{L B}-\mu_{4} A_{M} P, \\
& \alpha_{M} \frac{\mathrm{d} T_{M}}{\mathrm{~d} t}=m_{s} I A_{M}-m_{e},
\end{aligned}
$$




$$
\begin{aligned}
\frac{\mathrm{d} P}{\mathrm{~d} t} & =\mu_{a m p}-\mu_{4} A_{M} P-\mu_{3} R_{M} I P, \\
\alpha_{A} \frac{\mathrm{d} T_{A}}{\mathrm{~d} t}= & k_{a a} I A_{A} G_{A}-\frac{\beta_{f}}{1+k_{f t} I^{2}}, \\
\alpha_{A} \frac{\mathrm{d} A_{A}}{\mathrm{~d} t}= & -3 k_{a a} I A_{A} G_{A}+3 k_{a}\left(1+k_{a i} I\right)\left(1-k_{l p}\right) T_{C B}+3 k_{b a} T_{L B}+k_{n a} A_{N B}, \\
\alpha_{A} \frac{\mathrm{d} L_{A}}{\mathrm{~d} t}= & \frac{\beta_{f}}{1+k_{f t} I^{2}}-k_{g p} L_{A}, \\
\alpha_{A} \frac{\mathrm{d} G_{A}}{\mathrm{~d} t}= & d_{B A}\left(1+k_{g a} I\right)\left(G_{B}-G_{A}\right)-k_{a a} I A_{A} G_{A}, \\
\frac{\mathrm{d} T_{C B}}{\mathrm{~d} t}= & S_{F}(t)-k_{c m} T_{C B}-k_{c l} T_{C B}-k_{a}\left(1+k_{a i} I\right) T_{C B}, \\
\frac{\mathrm{d} A_{N B}}{\mathrm{~d} t}= & -k_{b m} A_{N B}-k_{b l} A_{N B}-k_{n a} A_{N B}+\frac{3 \beta_{f}}{1+k_{f t} I^{2}}+3 k_{a} k_{l p}\left(1+k_{a i} I\right) T_{C B}, \\
\frac{\mathrm{d} T_{L B}}{\mathrm{~d} t}= & F(I) \frac{v_{9} T_{L}}{k_{9}+T_{L}}+k_{9 a} S_{L}-k_{r} T_{L B}-k_{t} T_{L B}-k_{b a} T_{L B}, \\
\frac{\mathrm{d} G_{B}}{\mathrm{~d} t}= & k_{g l} G_{L}-k_{g l 2} G_{B}-\left(1+k_{g i} I\right)\left(k_{g m} G_{B}-k_{g m 2} G_{M}\right) \\
& -d_{B A}\left(1+k_{g a} I\right)\left(G_{B}-G_{A}\right)-\mu_{1} .
\end{aligned}
$$

\subsection{List of Parameters}

Table 2 summarises the expected fasted steady-state values of all the system's variables. The values used for the parameters, and source for these values are quoted in Table 3. Following these tables, more detail is given on the derivations of a couple of the parameter values which are more difficult to obtain $\left(v_{9}, k_{b a}, k_{b k}\right)$. 


\begin{tabular}{lcll}
\hline Description & Variable & Steady value & Reference \\
\hline Liver Glucose & $G_{L}$ & $8 \mathrm{mmol} / l$ & {$[31]$} \\
Liver Glycogen & $Y_{L}$ & $50 \mathrm{mmol} / l$ & Healthy fasting liver conc. \\
Liver G-6-P & $P_{L}$ & $2.06 \mathrm{mmol} / l$ & Model run to steady state. \\
Liver Pyruvate & $R_{L}$ & $0.37 \mathrm{mmol} / l$ & {$[31]$} \\
Liver FFA & $A_{L}$ & $0.57 \mathrm{mmol} / l$ & {$[31]$} \\
Liver TAG Storage Pool & $T_{L}$ & $40 \mathrm{mmol} / l$ & Healthy fasting liver conc. \\
Liver TAG Secretory Pool & $S_{L}$ & $0.0149 \mathrm{mmol} / l$ & Model run to steady state. \\
\hline Muscle Glucose & $G_{M}$ & $0.5 \mathrm{mmol} / l$ & {$[36]$} \\
Muscle Glycogen & $Y_{M}$ & $20 \mathrm{mmol} / l$ & Healthy fasting muscle conc. \\
Muscle G-6-P & $P_{M}$ & $0.133 \mathrm{mmol} / l$ & {$[48]$} \\
Muscle Pyruvate & $R_{M}$ & $0.009 \mathrm{mmol} / l$ & {$[36]$} \\
Muscle FFA & $A_{M}$ & $0.53 \mathrm{mmol} / l$ & {$[36]$} \\
Muscle TAG & $T_{M}$ & $14.8 \mathrm{mmol} / l$ & {$[36]$} \\
\hline Plasma Glucose & $G_{B}$ & $5 \mathrm{mmol} / l$ & {$[19]$} \\
Plasma Insulin & $I$ & $60 \mathrm{pmol} / l$ & {$[19]$} \\
Plasma NEFA & $A_{N B}$ & $0.5 \mathrm{mmol} / l$ & {$[19]$} \\
Plasma Exogenous CM TAG & $T_{C B}$ & $0 \mathrm{mmol} / l$ & {$[19]$} \\
Plasma Endogenous LP TAG & $T_{L B}$ & $1 \mathrm{mmol} / l$ & {$[19]$} \\
\hline Adipose Glucose & $G_{A}$ & $2.53 \mathrm{mmol} / l$ & {$[56]$} \\
Adipose FFA & $A_{A}$ & $0.57 \mathrm{mmol} / l$ & {$[31]$} \\
Adipose TAG & $T_{A}$ & $500 \mathrm{mmol} / l$ & Healthy conc. \\
Adipose Glycerol & $L_{A}$ & $0.17 \mathrm{mmol} / l$ & {$[25]$} \\
& & & \\
& & &
\end{tabular}

Table 2: Steady state concentrations. 
Table 3: Parameter values used in simulations.

\begin{tabular}{|c|c|c|c|}
\hline Parameter & Value & Description & Source \\
\hline$\overline{\alpha_{A}}$ & $15.6 l$ & Volume of adipose tissue & $\begin{array}{l}\text { A } 70 \mathrm{~kg} \text { individual with a BMI of } 21.5 \mathrm{~kg} / \mathrm{m}^{2} \text { (healthy), has an estimated } \\
\text { body fat of } 20 \% \text { ( } 1994 \text { National Health and Nutrition Examination Study). } \\
\text { Conversion to volume is obtained from an adipose tissue density of } 0.9 \mathrm{~kg} / \mathrm{l} \text {. }\end{array}$ \\
\hline$\alpha_{B}$ & $5 l$ & Volume of blood & $19]$. \\
\hline$\alpha_{L}$ & $1.6 l$ & Volume of the liver & 51. \\
\hline$\alpha_{M}$ & $26.4 l$ & Volume of skeletal muscle & $\begin{array}{l}\text { A } 70 \mathrm{~kg} \text { individual has an estimated } 40 \% \text { body weight in skeletal muscle. } \\
\text { Conversion to volume is obtained from an skeletal muscle tissue density of } \\
1.06 \mathrm{~kg} / \mathrm{l} \text {. }\end{array}$ \\
\hline$\beta_{f}$ & $0.117 \mathrm{mmol} / \mathrm{min}$ & Adipose release of TAG to NEFA & Approximated from data in Bickerton [8, 9]. \\
\hline$\beta_{l}$ & $12 l / \min$ & Liver glycogenolysis & $\begin{array}{l}\text { Approximations from considering data in Taylor } 55 \text {, solving glycogenolysis } \\
\text { and glycogenesis rate equations simultaneously. }\end{array}$ \\
\hline$\beta_{m}$ & $82.5 l / \min$ & Muscle glycogenolysis & 31. \\
\hline$\mu_{a m p}$ & 1.8 & Notional AMP/ADP creation rate & $\begin{array}{l}\text { A typical energy expenditure of } 10 M J / d a y \text { is assumed } 19] .15 \% \text { of this is } \\
\text { satisfied by protein consumption }[19] \text {, and } 2025 \text { from brain usage, leaving } \\
6475 \mathrm{kj} \text {. Conversion for molar density and energy density gives } 1.8 \text {. }\end{array}$ \\
\hline$\mu_{b}$ & $0.133 \mathrm{mmol} / \mathrm{min}$ & Lactate production by red blood cells & $19]$. \\
\hline$\mu_{s}$ & $7.19 \times 10^{6} \mathrm{lmmol}^{-1} \mathrm{~min}^{-1}$ & Muscle FFA esterification to TAG, & Rate chosen such that the skeletal muscle FFA concentration is $0.57 \mathrm{mmol} / \mathrm{l}$ \\
\hline$\mu_{1}$ & $0.588 \mathrm{mmol} / \mathrm{min}$ & Plasma glucose usage & $\begin{array}{l}\text { Total rate is the sum of brain and red blood cell use. Brain usage of } \\
\text { plasma glucose is } 0.458 \mathrm{mmol} / \mathrm{min}(120 \mathrm{~g} / \text { day }) \text {. Red blood cell usage } 0.133 \\
(25 \mathrm{mg} / \mathrm{min}) 19 .\end{array}$ \\
\hline$\mu_{3}$ & $7.839 \times 10^{7} \mathrm{lmmol}^{-1} \mathrm{~min}^{-1}$ & Muscle G-6-P usage & $\begin{array}{l}\text { Approximate glucose usage in periphery is } 3.2 M J 19 . \text { Rate takes into ac- } \\
\text { count G-6-P and insulin concentration, and FFA usage. Ratio of } \mu_{3} \text { and } \mu_{4} \\
\text { determines usage. }\end{array}$ \\
\hline$\mu_{4}$ & $100 l / \min$ & Muscle FFA usage & $\begin{array}{l}\text { Approximate FFA usage in periphery is } 6.1 M J[19] \text {. Rate takes into account } \\
\text { muscle concentration, and glucose usage. Ratio of } \mu_{3} \text { and } \mu_{4} \text { determines } \\
\text { usage. }\end{array}$ \\
\hline$c_{0}$ & $0.1 \mathrm{mmol} / \mathrm{l}$ & Small parameters & Chosen to be $<O(0.01)$ of glycogen concentration \\
\hline$c c$ & $2.5 \mathrm{mmol} / \mathrm{l}$ & $\begin{array}{l}\text { Range of glucose concentrations over } \\
\text { which excess insulin secretion occurs }\end{array}$ & $\begin{array}{l}\text { Obtained from a simple least squares data fit of experiments in 22] (Figure } \\
\text { 1A). }\end{array}$ \\
\hline
\end{tabular}




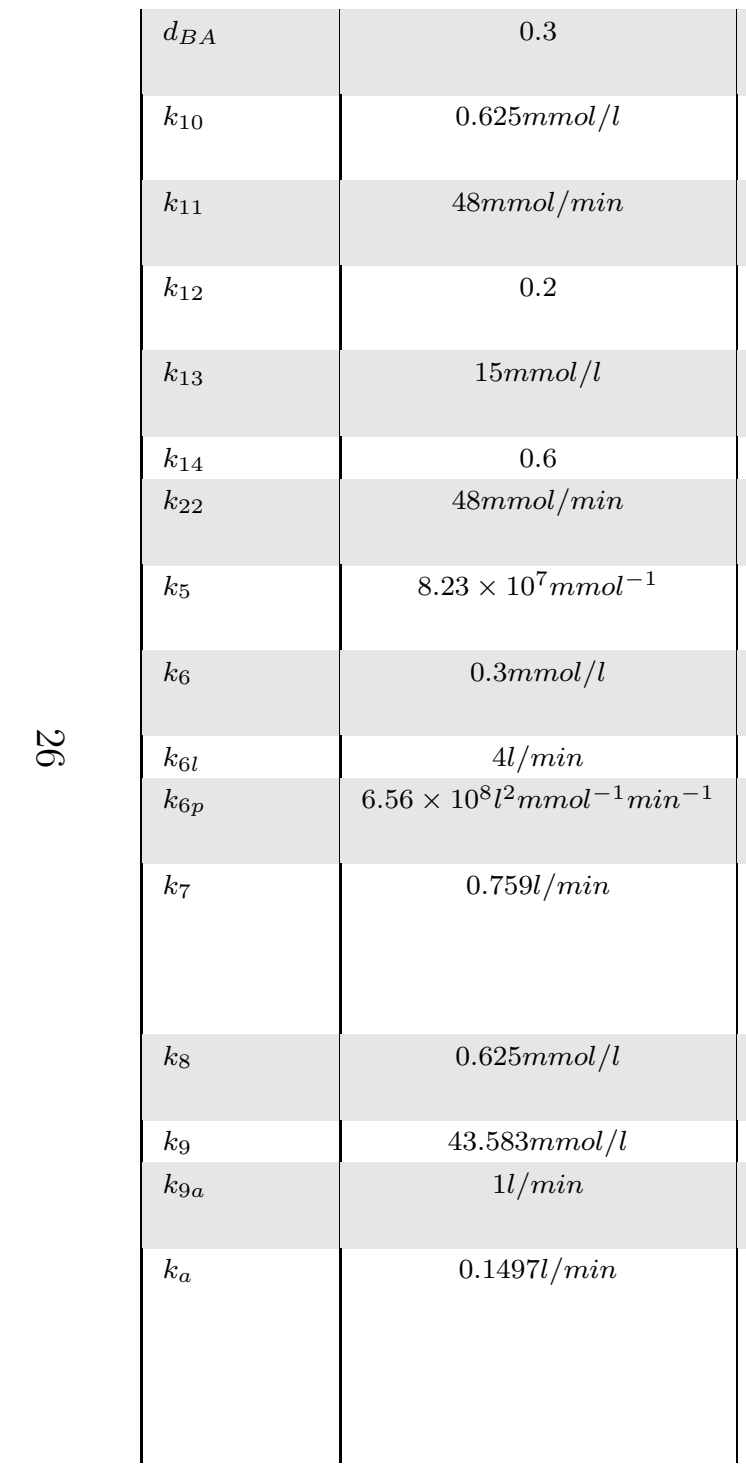

Adipose uptake of glucose

Affinity for hydrolysis of TAG to secretory pool

Basal insulin secretion rate

Increased fraction of VLDL1 secretion by insulin

Rate at which insulin modifies the fraction of VLDL1/VLDL2 secretion

Basal VLDL1 secretion fraction

Excess insulin secretion rate due to glucose stimulation

Flux control coefficient for insulin inhibition of FFA oxidation

Affinity for VLDL2 TAG secretion through secretory pathway

Liver glucose dephosphorylation rate

Muscle G-6-P to pyruvate conversion rate

Max rate of FFA oxidation

Affinity for esterification of FFA to TAG

Affinity of additional bulk lipidation Release of VLDL from secretory pathway

Adipose FFA uptake of CM TAG (insulin independent)
Rate chosen such that the adipose glucose steady state concentration is $2.54 \mathrm{mmol} / \mathrm{l}$ [56].

Arbitrary small parameter. Chosen to be $<O(0.01)$ of TAG pool.

Obtained from a simple least squares data fit of experiments in 22] (Figure 1A).

Simple least squares fitting of multiple data sets [3] [4] 39] 20.

See $k_{12}$

See $k_{12}$

Obtained from a simple least squares data fit of experiments in 22] (Figure $1 \mathrm{~A})$

Estimated from experiments in Westerbacka 60.

Chosen to be $<O(0.01)$ of FFA concentration

\section{1}

Chosen such that steady state concentration of muscle G-6-P is $0.13 \mathrm{mmol} / \mathrm{l}$.

For a liver TAG concentration of $45 \mathrm{mmol} / \mathrm{l}$ at steady state, the oxidation rate is set to maintain this. Parameter is adjusted for insulin inhibition by fitting to experimental data in Westerbacka et al 60]. Note, we assume ketone production is proportional to FFA oxidation.

Small parameter chosen to be $<O(0.1)$ of FFA concentration.

See $v_{9}$.

Set to unity.

Turnover of chylomicron TAG is estimated to have a half life of $4.5 \mathrm{~min}$ 21].

We use a 5 minute half life giving a total degradation rate of $\log 2 / 5=0.1386$. The liver takes up gives $4 \%$ of this 23]. Bickerton 8 shows adipose tissue uptake 3 times more than skeletal muscle, therefore adipose uptake given by $72 \%$ of 0.1386 . 


\begin{tabular}{|c|c|c|c|}
\hline & $k_{a a}$ & $3.11 \times 10^{5} l^{2} \mathrm{mmol}^{-2} \mathrm{~min}^{-1}$ & Adipose FFA esterification to TAG \\
\hline \multirow{18}{*}{$\underset{v}{N}$} & $k_{a i}$ & $2.08 \times 10^{6} \mathrm{mmol}^{-1}$ & $\begin{array}{l}\text { Adipose FFA uptake of CM TAG (in- } \\
\text { sulin dependent) }\end{array}$ \\
\hline & $k_{a l}$ & $0.00002 l^{2} \mathrm{mmol}^{-1} \mathrm{~min}^{-1}$ & Pyruvate to $\mathrm{ACoA}$ conversion rate \\
\hline & $k_{b a}$ & $0.0104 l / \min$ & $\begin{array}{l}\text { Adipose uptake of endogenous LP } \\
\text { TAG }\end{array}$ \\
\hline & $k_{b l}$ & $0.156 l / \min$ & Liver uptake of plasma NEFA \\
\hline & $k_{b m}$ & $0.226 l / \min$ & Muscle uptake of plasma NEFA \\
\hline & $k_{c l}$ & $0.0075 \mathrm{l} / \mathrm{min}$ & $\begin{array}{l}\text { Liver FFA Take-Up of chylomicron } \\
\text { TAG }\end{array}$ \\
\hline & $k_{c m}$ & $0.0449 l / \mathrm{min}$ & $\begin{array}{l}\text { Muscle FFA take up of chylomicron } \\
\text { TAG }\end{array}$ \\
\hline & $k_{d}$ & $1.733 \times 10^{8} \mathrm{l} / \mathrm{min}$ & Insulin degradation rate \\
\hline & $k_{d l}$ & $3.5 \times 10^{8} \mathrm{l} / \mathrm{mmol}$ & $\begin{array}{l}\text { Liver glycogenolysis (insulin inhibited } \\
\text { rate) }\end{array}$ \\
\hline & $k_{d y}$ & $4 \times 10^{8} \mathrm{mmol}^{-1}$ & $\begin{array}{l}\text { Muscle glycogenolysis (insulin inhib- } \\
\text { ited rate) }\end{array}$ \\
\hline & $k_{f t}$ & $1.67 \times 10^{14} \mathrm{mmol}^{-1}$ & $\begin{array}{l}\text { Adipose release of TAG to NEFA (in- } \\
\text { sulin inhibited rate) }\end{array}$ \\
\hline & $k_{g a}$ & $1.67 \times 10^{6} \mathrm{mmol}^{-1}$ & Adipose uptake of glucose \\
\hline & $k_{g i}$ & $2.632 \times 10^{7} \mathrm{mmol}^{-1}$ & $\begin{array}{l}\text { Approximated using data from Kelley } \\
\text { and Kraegen simultaneously } 30,35]\end{array}$ \\
\hline & $k_{g l}$ & $0.9277 l / \min$ & Liver to blood glucose scalar \\
\hline & $k_{g l 2}$ & $0.396 l / \min$ & Blood to liver glucose scalar \\
\hline & $k_{g m}$ & $0.0380 \mathrm{l} / \mathrm{min}$ & Blood to muscle glucose scalar \\
\hline & $k_{g m 2}$ & $0.0380 l / \min$ & Muscle to blood glucose scalar \\
\hline & $k_{g p}$ & $0.311 l / \min$ & Adipose glycerol return rate (to liver) \\
\hline
\end{tabular}

Rate chosen such that the adipose FFA concentration is $0.57 \mathrm{mmol} / \mathrm{l}$ in the steady state.

Negligible compared to insulin independent take up.

\section{1], 32].}

\section{See description below}

See description below

See $k_{b l}$, muscle $50 \%$ of (of 0.4510 )

See $k_{a}$ Rate given by $4 \%$ of 0.1386 .

See $k_{a}$.Rate given by $24 \%$ of 0.1386 .

The half life of insulin is approximately $4-6$ minutes 15 . The degradation rate is calculated by (ln $2 /$ half life). We use the lower bound of 4 minutes.

See $\beta_{l}$

See $\beta_{m}$

Approximated from data in Bickerton [8, 9].

Rate chosen such that the adipose glucose steady state concentration is $2.54 \mathrm{mmol} / \mathrm{l}[\underline{56}$.

See $k_{g}$

In combination with $k_{g l 2}$ this confers steady state concentrations of liver $(8 \mathrm{mmol} / \mathrm{l})$ to blood $(5 \mathrm{mmol} / \mathrm{l})$

In combination with $k_{g l}$ this confers steady state concentrations of liver $(8 \mathrm{mmol} / \mathrm{l})$ to blood $(5 \mathrm{mmol} / \mathrm{l})$

In combination with $k_{g m 2}$ this confers steady state concentrations of muscle $(0.5 \mathrm{mmol} / \mathrm{l})$ to blood $(5 \mathrm{mmol} / \mathrm{l})$.

In combination with $k_{g m}$ this confers steady state concentrations of muscle $(0.5 \mathrm{mmol} / \mathrm{l})$ to blood $(5 \mathrm{mmol} / \mathrm{l})$.

Rate chosen such that the glycerol steady state concentration is $0.17 \mathrm{mmol} / \mathrm{l}$ 


\begin{tabular}{|c|c|}
\hline$k_{l p}$ & 0.25 \\
\hline$k_{L G}$ & $8.95 \mathrm{mmol} / \mathrm{l}$ \\
\hline$k_{L H}$ & $0.0115 \mathrm{mmol} / \mathrm{l}$ \\
\hline$k_{M H}$ & $8.95 \mathrm{mmol} / \mathrm{l}$ \\
\hline$k_{n a}$ & 0.0697 \\
\hline$k_{p}$ & $1.41 \times 10^{7} l^{2} \mathrm{mmol}^{-1} \mathrm{~min}^{-1}$ \\
\hline$k_{p 6}$ & $1.93 \times 10^{8} \mathrm{mmol}^{-1}$ \\
\hline$k_{p p}$ & $0.5 l / \min$ \\
\hline$k_{r}$ & $0.00058 \mathrm{l} / \mathrm{min}$ \\
\hline$k_{r e p}$ & $2.98 \mathrm{l} / \mathrm{mmol}$ \\
\hline$k_{t}$ & $0.00348 \mathrm{l} / \mathrm{min}$ \\
\hline$k_{y l}$ & $1.28 \times 10^{6} l^{2} \mathrm{mmol}^{-1} \mathrm{~min}^{-1}$ \\
\hline$k_{y m}$ & $21.364 l^{2} \mathrm{mmol}^{-1} \mathrm{~min}^{-1}$ \\
\hline$l_{\max }$ & $400 \mathrm{mmol} / \mathrm{l}$ \\
\hline$m_{\max }$ & $100 \mathrm{mmol} / \mathrm{l}$ \\
\hline$v$ & $7 \mathrm{mmol} / \mathrm{l}$ \\
\hline$v_{10}$ & $0.1 l / \min$ \\
\hline$v_{12}$ & $40 \mathrm{mmol} / \mathrm{l}$ \\
\hline$v_{6}$ & $0.0158 l / \min$ \\
\hline$v_{8}$ & $0.333 l / \min$ \\
\hline$v_{9}$ & $0.0159 l / \min$ \\
\hline$v_{L G}$ & $14.3 l / \mathrm{min}$ \\
\hline
\end{tabular}

Proportion of TAG released into the plasma from the action of LPL.

Glucokinase affinity coefficient

Hexokinase affinity coefficient

Hexokinase affinity coefficient

Adipose uptake of plasma NEFA

Pyruvate to ACoA conversion rate

Liver pyruvate to G-6-P (insulin inhibited)

Muscle Pyruvate return to liver pyruvate

Liver uptake of endogenous LP TAG

Hexokinase inhibition by G-6-P

Muscle uptake of endogenous LP TAG

Liver glycogenesis rate

Muscle glycogenesis rate

Maximum liver glycogen concentration

Maximum muscle glycogen concentra-

tion

Required glucose concentration for half maximal secretion of insulin

Max rate of hydrolysis of TAG to secretory pool

Half maximal concentration for insulin's effect on switching the secretion of lipoprotein fractions

Max rate of VLDL2 TAG secretion through secretory pathway

Max rate of FFA esterification to TAG

Maximum rate for the bulk lipidation of VLDL

Glucokinase max rate
Estimated

53. Note, mouse data is used here

53. Note, mouse data is used here.

Same as $k_{L G}$

See $k_{b l}$, adipose $15.45 \%$ of (of 0.4510 )

31,32 .

Set such that gluconeogenesis and glycogenolysis produce equal quantities of glucose [59].

Rate chosen such that the muscle pyruvate concentration is at the given steady state.

See $k_{b a}$. Rate given by $4 \%$ of 0.0262 .

Simple least squares fitting of experiments in Crane 10].

See $k_{b a}$. Rate given by $24 \%$ of 0.0262 .

Average of estimates in Price [48] and Shulman [51]. Rate is adjusted for glucose and insulin concentrations.

$55]$.

$19]$.

Obtained from a simple least squares data fit of experiments in 22 (Figure $1 \mathrm{~A})$

Set to release the same quantity of TAG as secreted in nascent VLDL particles.

See $k_{12}$

See $v_{9}$ description.

Comparatively very small, set to zero.

See additional parameter descriptions below.

Estimated rate from Sreenan 53|, Scaled for $1.6 l$ liver volume. Note, mouse data is used here. 


$\left|\begin{array}{l}v_{L H} \\ v_{M H} \\ y_{0}\end{array}\right|$

$5.57 l / \min$

$54.288 \mathrm{l} / \mathrm{min}$

$0.1 \mathrm{mmol} / \mathrm{l}$
Hexokinase max rate

Hexokinase max rate

Small parameters
Estimated rate from Sreenan 53|, Scaled for $1.6 l$ liver volume. Note, mouse data is used here.

See $v_{L H}$. Scaled for $16.4 l$ muscle volume.

Chosen to be $<O(0.01)$ of glycogen concentration. 
$v_{9}$ : Calculations from experimental data in 3, 2, 4]. For adjusted model parameters, we calculate the average TAG per apoB molecule in VLDL2, $27.5 \mathrm{mg} \mathrm{kg}^{-1} \mathrm{day}^{-1}$ / $2.1 \mathrm{mg} \mathrm{kg}^{-1} \mathrm{day}^{-1}=13.095$, multiply by total number of apoB secreted $9.2 \mathrm{mgkg}^{-1} \mathrm{day}^{-1}$, which gives total TAG production excluding bulk lipidation from cytosolic TAG. Subtracting $13.095 \times 9.2$ (average TAG per apoB multiplied by total apoB secretion), from the VLDL1 TAG production, gives bulk lipidation. As a function of liver fat, we perform a simple non-linear least squares fit data to model equations; $V_{\max }$ and $K_{M}$ follow. Note $1 \mathrm{~g}$ of TAG is taken to be equal to $1.14 \mathrm{mmol}$ in these calculations.

$k_{b a}$ : Total rate of uptake of VLDL-TAG is set at 0.0262 to maintain a fasting plasma TAG of $1 \mathrm{mmol} / \mathrm{l}$. Hodson et al [23] estimate $4 \%$ of TAG is taken up from the remnant particles by the liver. Bickerton [8] estimates adipose tissue takes up 3 times more TAG than skeletal muscle. Thus adipose tissue takes up $72 \%$ of 0.0262 , whilst skeletal muscle takes up $24 \%$ of 0.0262 .

$k_{b l}$ : Plasma NEFA half life estimates vary from $30 \mathrm{~s}$ to $3 \mathrm{~min}$ [27], giving a total degradation rate of 1.3863 to $0.2310 \mathrm{~min}^{-1}$. We choose $0.4510 \mathrm{~min}^{-1}$ calculated from setting fasting NEFA to $0.5 \mathrm{mmol} / l$. Jelic [26] estimates that $34.55 \%$ of plasma NEFA is taken up by the liver. This value is in relatively good agreement with the work of Nielsen et al. [4], who use measurements of palmitate uptake in leg muscle.

This completes the parameterisation of the model. In the next section (\$3) we summarise results of short-time simulations, before moving on to test the model against independent data from longer timescale.

\section{Results}

A large body of experimental evidence is available for the profiles of plasma variables after a variety of single and mixed meals. For comparison, model simulations are presented alongside sample experimental data in section 3.1. As plasma is the main transport system between compartments, the concentrations of key carbohydrate and lipid molecules in the plasma gives confidence in the model for plasma and other tissues. 


\subsection{Plasma variables}

Cruz et al 11] simultaneously measure changes in plasma glucose, insulin, NEFA, and TAG, at multiple time points over an interval of 0 to 480 minutes in response to a single mixed meal. Meal composition is broken down as $52 \%$ fat, $40 \% \mathrm{CHO}$ and $8 \%$ protein. The time scales for absorption of metabolites, the parameters $\beta_{F}$ and $\beta_{G}$ in (11), are determined by peak absorption time after ingestion; 180 minutes for fat, and 35 minutes for carbohydrates. Figure 2 compares model simulations to experimental data.
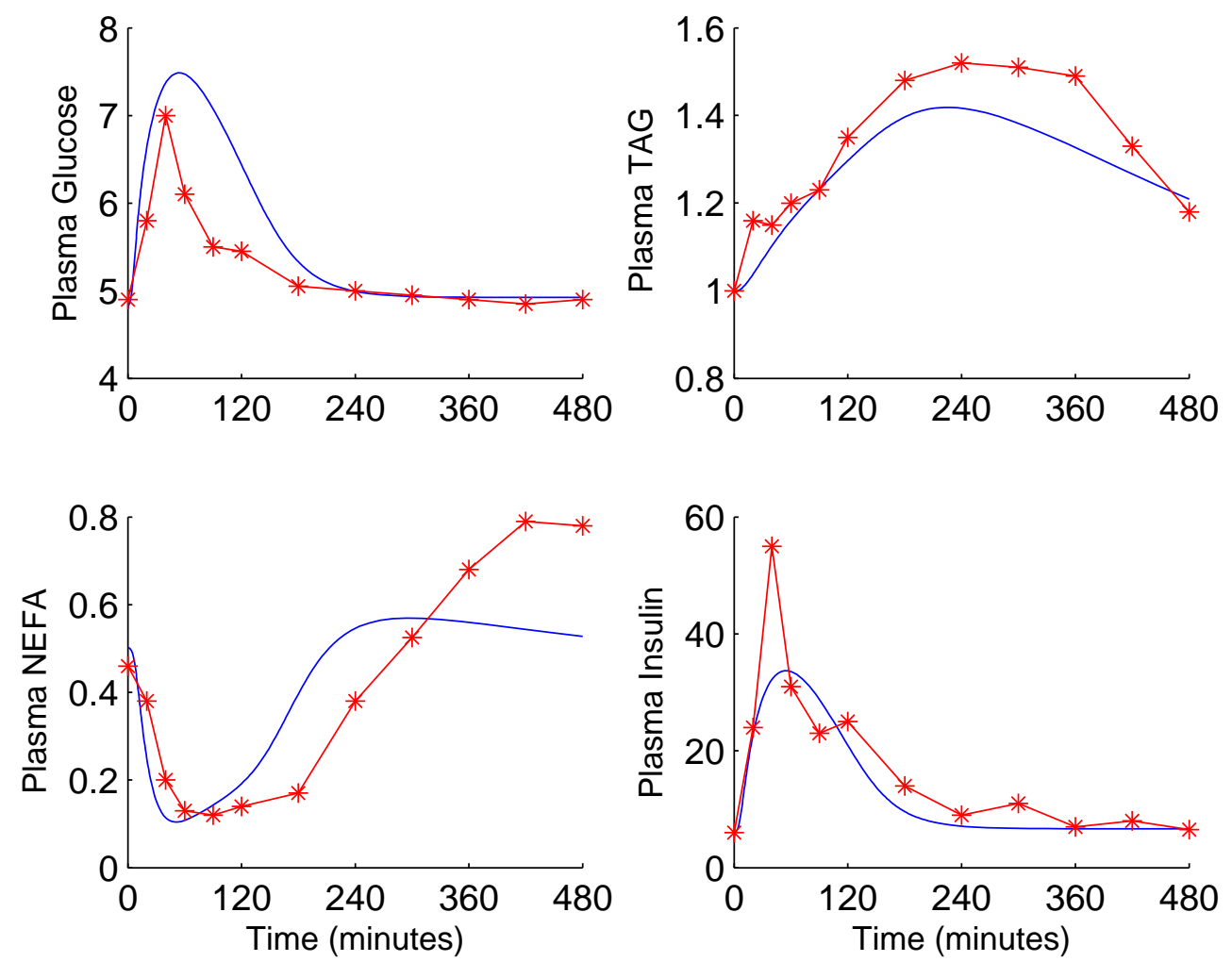

Figure 2: The evolution of plasma variables for a single meal compared to model simulations: Experimental data (red asterisk), model predictions (blue). All variables have units mmol/l, except insulin $\left(10^{-7} \mathrm{mmol} / \mathrm{l}\right)$.

Reasonable agreement is shown between experimental data and model simulations for both plasma glucose and insulin. Glucose rises after the mixed meal as it is absorbed from the gut, and a concomitant rise in plasma insulin is observed. Both metabolites 
return to near steady state over a time of 240 minutes. Qualitative agreement is shown between simulations and experimental data for plasma NEFA. Concentrations initially decrease in response to the mixed meal, the main change observed occurring in the inhibition of adipose tissue lipolysis. After insulin recovers, NEFA initially overshoots its steady state as the action of LPL on circulating chylomicrons leaks fatty acids into the plasma. The likely difference between our simulations and experimental data occurs due to the up-regulation of LPL in response to insulin occurs with a delay of about 4 hours which is not included in the model. As this is timed to occur when chylomicron concentrations are peaking in the system, the overshoot would be higher than predicted by our model. Had plasma NEFA been measured for longer in the experiment, we would expect it to return to steady state concentration within hours, in the same manner as our simulations.

\subsection{Nonplasma variables}

Variables for which we have no experimental data to compare against are plotted and discussed in the appendix Appendix A

\subsection{Comparison with 24 hour meal data}

To consider longer timescales, we begin by simulating a typical day with 3 meals. A different data set 24] (provided by the Oxford Lipid Metabolism group) is used for these longer experiments. Three isocaloric meals were fed at intervals of 5 hours apart, with frequent sampling of plasma insulin, glucose, NEFA and TAG. The carbohydrate and fat composition of each meal is summarised in Table 4 and complete experimental protocol for this data set is detailed in Hodson et al 24]. Experimental results are shown alongside model predictions for the 24 hour period in Figure 3.

\begin{tabular}{|l|l|l|l|}
\hline & Breakfast & Lunch & Dinner \\
\hline Carbohydrate & $108.2 \mathrm{~g}$ & $108.7 \mathrm{~g}$ & $106.2 \mathrm{~g}$ \\
of which sugars & $65.8 \mathrm{~g}$ & $24.7 \mathrm{~g}$ & $52.5 \mathrm{~g}$ \\
Fat & $36.0 \mathrm{~g}$ & $34.3 \mathrm{~g}$ & $33.1 \mathrm{~g}$ \\
\hline
\end{tabular}

Table 4: Meal composition 

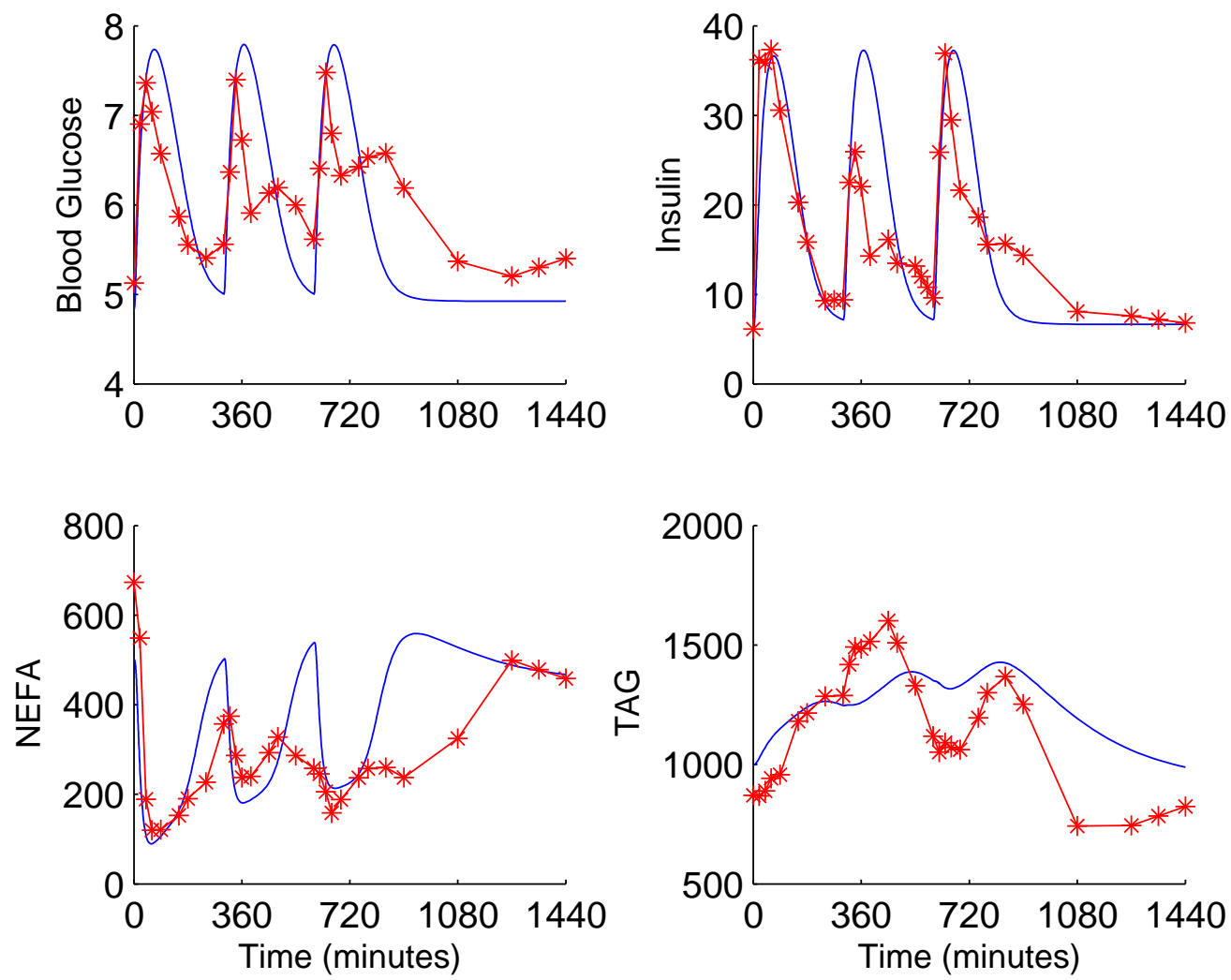

Figure 3: The evolution of plasma variables over 24 hours, meals given at times 0, 5, 10 hours. Experimental data (red asterisk), model predictions (blue). All variables have units mmol/l, except insulin $\left(10^{-7} \mathrm{mmol} / \mathrm{l}\right)$. 
Model simulations provide an excellent fit to the glucose and insulin experimental data during the first meal (0-300 minutes duration). The qualitative fit to glucose breaks down during the second and third meals where the model fails to capture an additional secondary spike of glucose, and a longer tail in the postprandial period. Additionally, there is a qualitative change in the peak insulin concentration at meal two not predicted by the model. This suggests that a model to accurately predict changes in metabolite concentrations over a longer-term would need some memory of earlier meals.

Despite the similarity in meal composition, both in terms of energy density and substrate composition, a vastly different response in plasma insulin is observed between the first and third meals compared to the second. However, similar responses in plasma glucose levels are observed in the three meals, suggesting that lower insulin concentrations were needed for effective disposal of plasma glucose in the second meal. The difference in the glucose/insulin response between meals can be contrasted better in the phase-plane diagram shown in Figure 4. The first meal produces the highest insulin spike, and whilst the other two meals produce similar glucose spikes, the second meal produces a significantly lower insulin spike, before plasma glucose returns to a slightly higher basal level. Following the third meal, the glucose returns to an even more elevated level, showing an increase in insensitivity to glucose/insulin by the body, through the day.

The model predicts NEFA well at early times, but the accuracy of fit to experimental data declines for successive meals. In particular, entering the fasting period after the third meal (approximately 6 hours after ingestion), the model predicts an overshoot in NEFA, whilst the experimental NEFA remains suppressed longer (approximately 11 hours after ingestion of meal). This is almost certainly due to plasma insulin remaining elevated above normal fasting values.

Experimental TAG concentrations during the first meal show reasonable agreement with the model. Again, the fit breaks down during the second meal. This could potentially be explained by a lack of LPL activation since 

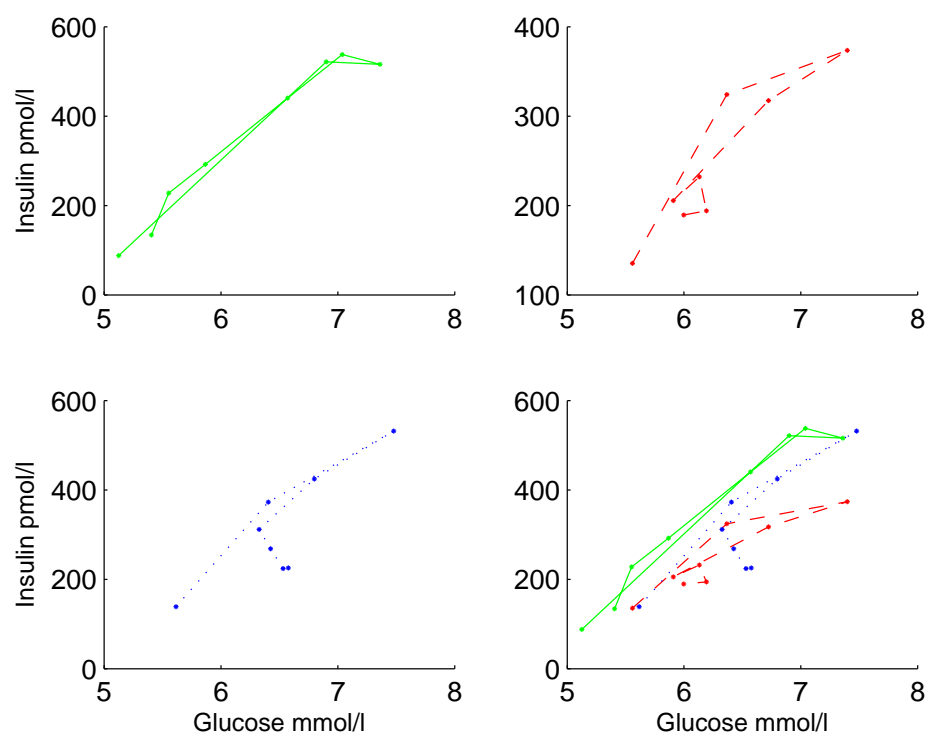

Figure 4: Insulin-glucose phase-plane for experimental data of mixed meals. Top left first meal (solid line), top right second meal (dashed line), bottom left third meal (dotted line), bottom right all meals

corresponding insulin concentrations are low, although other factors such as insulin regulated release of VLDL from the liver are likely to impact fit as well. Following the third meal, the decrease in TAG occurs at a rate much quick than is predicted by the model.

It is clear that the metabolic response to successive meals differs significantly to that of a single meal after a fast; something that the model fails to predict. Due to the strong influence insulin has throughout lipid metabolism, improved results would be obtained if we could reproduce the insulin response observed. Without additional modelling of insulin/glucose dynamics, we can predict the true effect of insulin on lipid metabolism by using the experimental insulin and glucose data as inputs to our model.

\subsection{Data fitting of 24 hours meal pattern}

We consider the accuracy of the model in predicting plasma NEFA and liver lipids using the experimentally derived glucose and insulin to infer the other variables. 
A cubic interpolating spline was fitted to the experimental for plasma glucose and insulin data (with ghost points of neighbouring splines at 270 minutes at 570 minutes), whilst all other variables remained the same (Figure 5). New simulations with spline approximations replacing equations (26) and (27) show better agreement with plasma NEFA (a major determinant of liver lipids). Under these conditions, hepatic TAG differs significantly in the fasted period (Figure 6), showing an emptying of lipid in the liver, rather than the build up of lipid seen in the initial simulation.
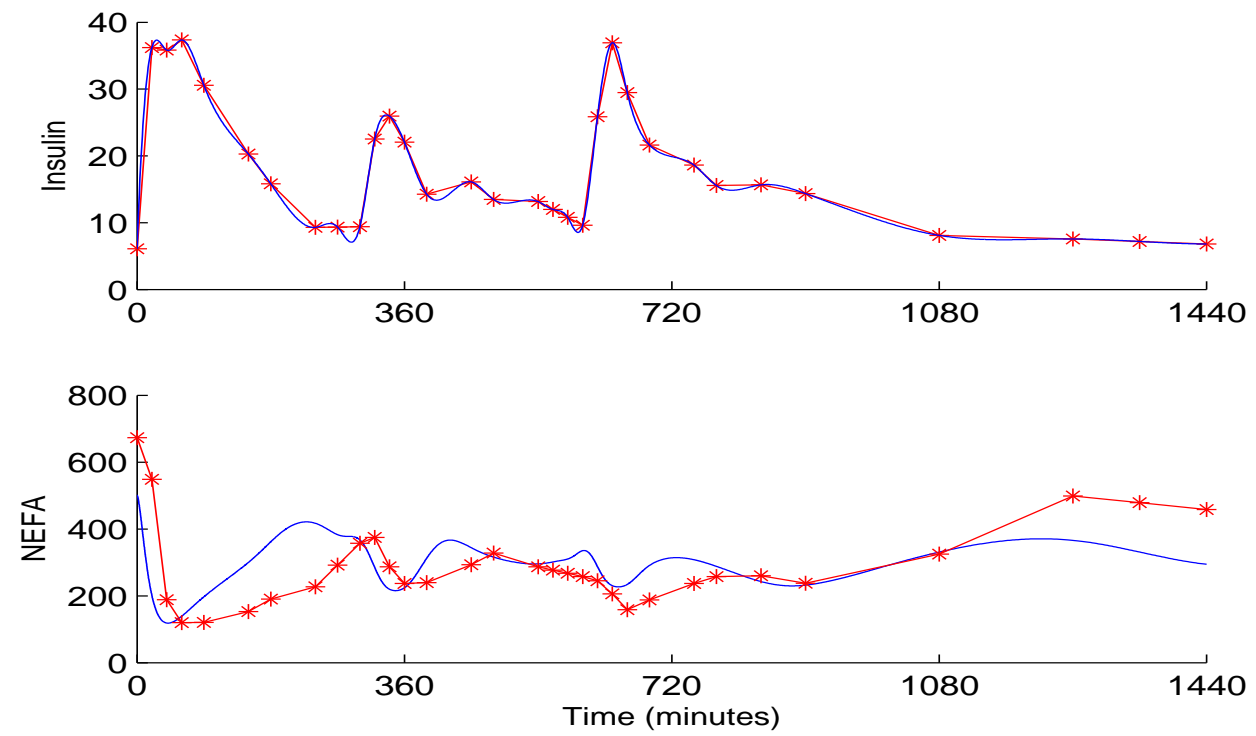

Figure 5: Top: Experimental forced-fit model of insulin concentration $I(t)$, Bottom: New prediction of plasma NEFA $A_{B}(t)$ plotted against time. Experimental data (red asterisk), forced-fit model predictions (blue line). 


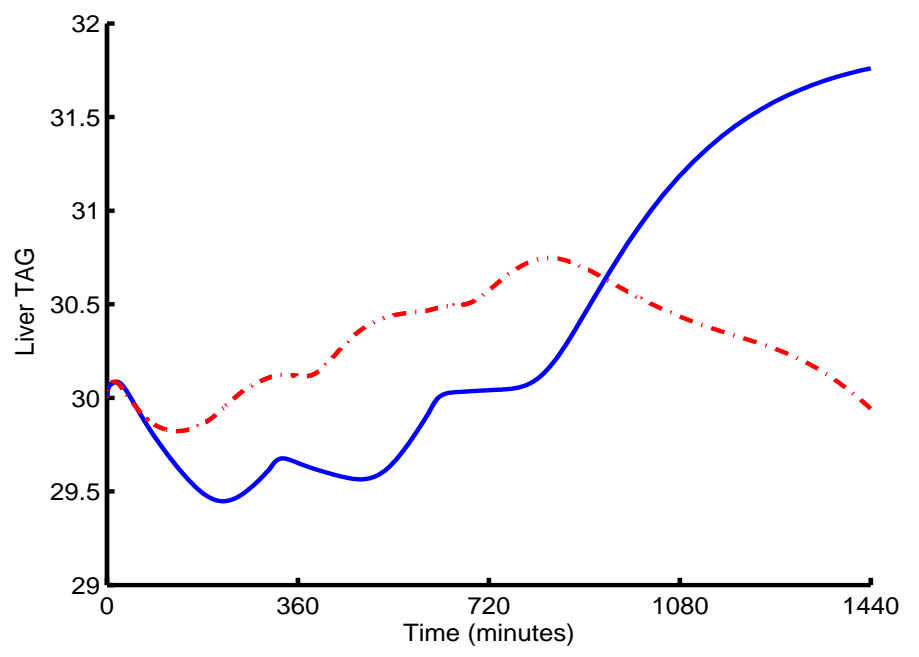

Figure 6: Comparison of initial simulation of hepatic TAG (solid blue line) with new comparison of hepatic TAG (dashed red line). 


\section{Discussion}

Our study of hepatic lipid metabolism has led us to produce a large compartmental ODE system covering multiple chemical species in multiple tissues. This has resulted in a system which is less detailed than many other single-tissue models available in the literature, but that integrates the non-hepatic tissues and pathways relevant to understanding the pathogenesis of hepatic steatosis.

Our initial single meal simulations show good agreement with experimental data, but the comparison with multiple meals is less accurate. The decreased insulin response in the second meal compared to the first could occur for a number of reasons. Although the amount of carbohydrate ingested is the same across all three meals, the ratio of simple to complex sugars varies. However, we have investigated this (analysis not shown here [4]]) and found that differences in the release rate of sugar from the gut do not account for the differences observed. This differing response could be explained by the first meal 'priming' the body to deal with carbohydrates, and so not requiring the same concentration of insulin to suppress plasma glucose. It may be speculated that, changes in insulin sensitivity after multiple meals provide the body with some 'long term memory' of previous metabolic events. However, the mechanisms underlying such an effect (should it actually exist) remain to be explored.

Simulations suggested that lower GI carbohydrate diets have the effect of reducing hepatic triglycerides, see Figure [7. In the model, this was achieved by staying in the postprandial-period longer than that of a high GI diet. This response is likely due to the nonlinear reduction in adipose free fatty acid release from the moderate hyperinsulinemia. In addition, model simulations suggested dietary composition has a significant impact on the amount of fat accumulated in the liver, with a higher percentage of fat in the diet leading to higher concentrations of TAG in the liver. This is due to both the increased delivery directly from chylomicrons and the associated NEFA spill-over, as well as the reduced suppression of NEFA release by adipose tissue from a lower insulin response.

The model suffers from several limitations. Protein interactions have been ignored, which, far from being separate from carbohydrate and fat metabolism, influence key 


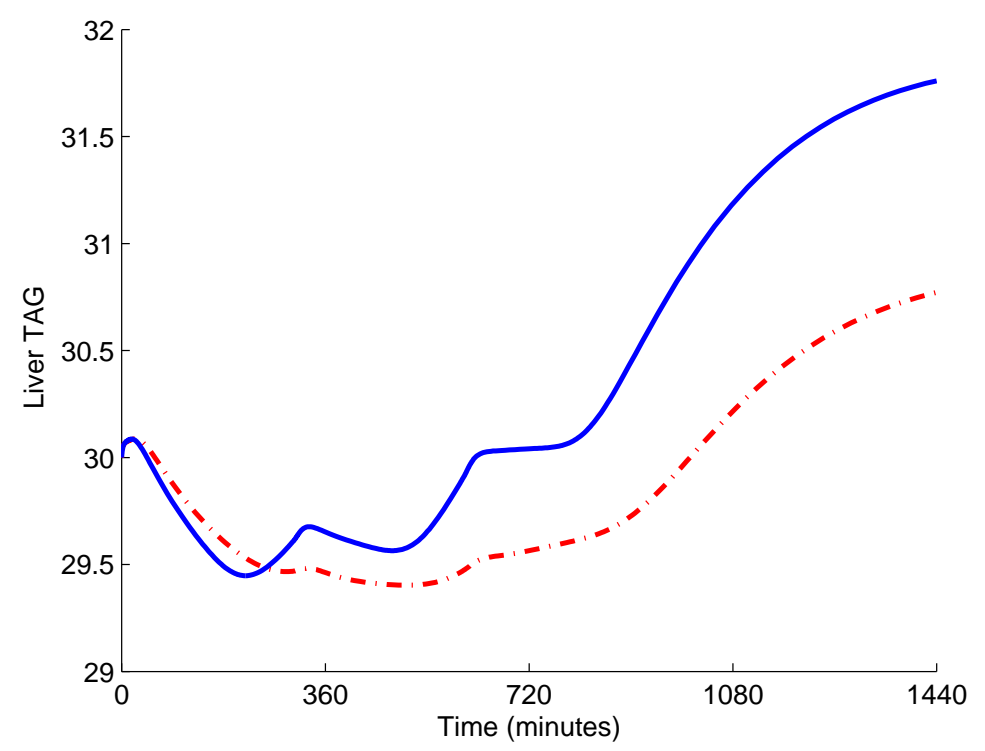

Figure 7: Hepatic TAG vs time for a 3 -meal high GI $\left(\beta_{G}=60\right)$ and low $\left(\beta_{G}=80\right)$ GI diet.

processes through the production of pyruvate from protein precursors (e.g. lactate and alanine). Absorption of carbohydrates and fat by the gut is known to be affected by the quantity of protein ingested. We have assumed a constant energy expenditure which may be unrealistic given the activity levels vary significantly over 24 hours. This may in part explain why our simulations for hepatic TAG show qualitatively different behaviour between the first 16 hours, and the last 8 hours. We have assumed processes that are up-regulated by glucagon are down-regulated by insulin and vice versa. In reality, high concentrations of insulin are not always matched by low levels of glucagon. Glucagon concentrations were not measured in the three meal data. Diet induced thermogenesis has been ignored, so energy consumption in the processing of carbohydrates and fats has not been fully taken into account. Intermediary metabolites such as those in the glycolytic pathway have been ignored, as non-fasting data is difficult to obtain, and they are not of primary concern to the model. As the composition of sugars was not available in our experimental data, we have assumed all carbohydrates are broken down to glucose. Had we factored in that a (small) proportion of these carbohydrates would be fructose, the insulin and hepatic TAG responses would have differed. Finally, we accepted that a delay of 4 hours in the activation of LPL would produce a better fit of the NEFA component of the 
model, however, we choose not to include this as the introduction of delay-differential equations would hinder future analysis.

More data is required to validate our model. We have relied upon simultaneous measurements of plasma TAG and plasma NEFA as an indicator of uptake and release rates of hepatic lipid, to infer net change in concentration. However, since DNL and beta-oxidation were not measured, we are missing the complete information required to infer changes in hepatic TAG indirectly. Frequent NMR imaging of hepatic TAG would provide a direct method to validate liver fat changes in response to diet. Combined with frequent samples of plasma metabolites, this would provide ideal data to validate our model.

The model we have created does not describe every process of energy metabolism in detail, it is rather an attempt to describe the factors that effect triglyceride accumulation in the liver using physiological mechanisms. It therefore provides a framework to study these mechanisms that are likely involved in the pathogenesis of hepatic steatosis. Given that insulin resistance is the most reproducible factor in NAFLD, in future work we intend to simulate varying degrees of resistance in single and multiple tissues in this model to investigate likely disease progression.

The results here emphasise the difficulty of predicting fat metabolism without a strong understanding of insulin; therefore, the interaction of carbohydrates and lipids through digestion and absorption to storage and oxidation in the body is relevant. The importance of meal timing has also been shown; our own simulations indicate an interaction when the body is not allowed to return to the fasting steady state before a new meal is fed, however the experimental data has shown this to be a far greater deviation than previously predicted.

The work here provides a framework to study the effects of insulin resistance in multiple tissues on the accumulation of TAG in the liver. Whilst the plasma behaviour could be reproduced with a smaller number of equations, this larger system incorporates the detail required to study whole body effects from singular processes within individual tissues. We intend to study the parameter adaptations required in the 
transition from healthy to insulin resistant states, and whether this is necessary for the increased deposition of liver fat seen in individuals with hepatic steatosis.

\section{Acknowledgements}

AP is grateful to MRC for funding. We thank Fredrik Karpe and Leanne Hodson for useful discussions and making data available to us.

\section{Appendix A. Supplementary graphs}

Variables for which we have no experimental data to compare against are illustrated for a complete day in which three identical mixed meals are simulated, five hours apart for a typical western diet. Each meal comprises $105 \mathrm{~g}$ carbohydrate (absorption rate $\beta_{G}=\beta_{F}=45$ minutes in (11) with $\alpha_{G}+\alpha_{F}=105 \mathrm{~g} / \beta^{2}$ ) and $35 \mathrm{~g}$ fat (absorption rate $\beta_{T}=180$ minutes, with $\alpha_{T}=35 \mathrm{~g} / \beta_{T}^{2}$ ). Model predictions for variables are shown in figures A.8, A.9, and A.10, Of particular interest is the observation that hepatic TAG initially decreases following a fatty meal. One explanation for this is that, since fat is ingested alongside carbohydrates in a mixed meal, secretion of insulin suppresses adipose lipolysis to a greater extent than hepatic TAG secretion is suppressed. Whilst this is an oversimplification, given the number of pathways affecting hepatic TAG concentration, a large quantity of carbohydrates can almost completely shut off adipose lipolysis (95\% reduction), reducing plasma NEFA (and therefore hepatic uptake) substantially. 

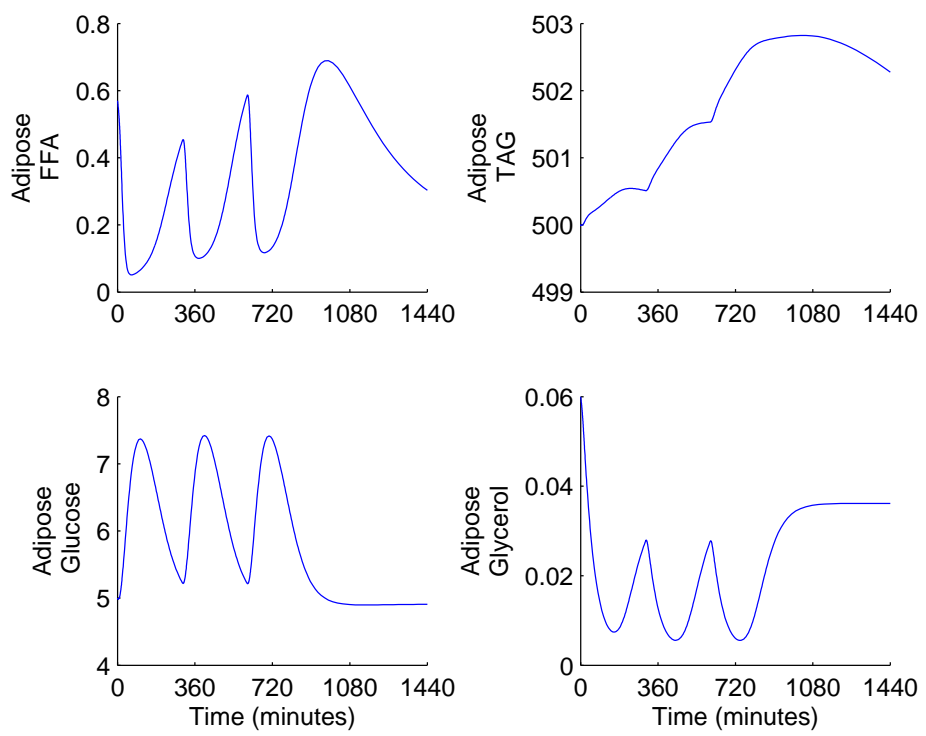

Figure A.8: The evolution of adipose associated variables over 24 hours, meals given at times 0,5, 10 hours. First column $A_{A}, G_{A}$. Second column $T_{A}, L_{A}$. All variables have units mmol/l.
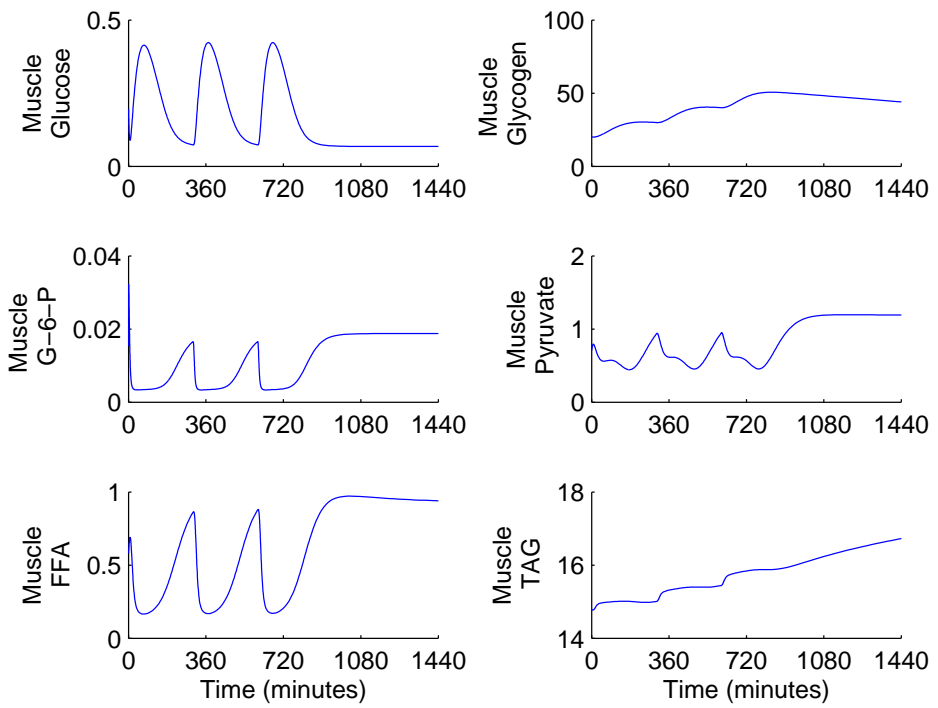

Figure A.9: The evolution of muscle associated variables over 24 hours, meals given at times 0, 5, 10 hours. First column $G_{M}, P_{M}, A_{M}$. Second column $Y_{M}, R_{M}, T_{M}$. All variables have units mmol/l. 

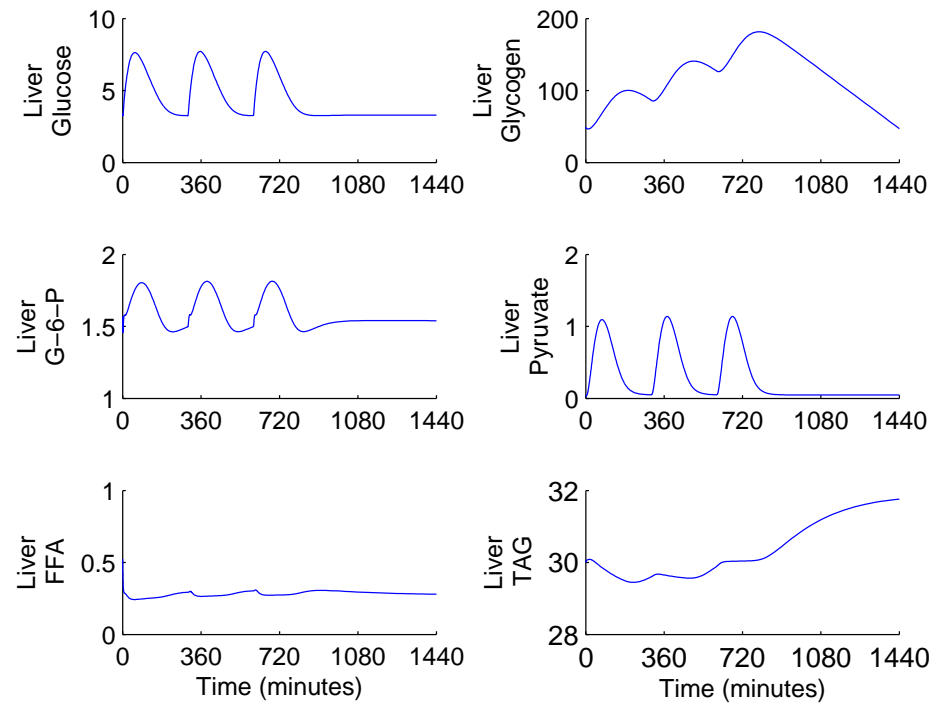

Figure A.10: The evolution of liver associated variables over 24 hours, meals given at times $0,5,10$ hours. First column $G_{L}, P_{L}, A_{L}$. Second column $Y_{L}, R_{L}, T_{L}$. All variables have units mmol/l. 


\section{References}

[1] M. Abramowitz and I.A. Stegun. Handbook of Mathematical Functions. Dover Publications, New York, 1964.

[2] M. Adiels, C. Packard, M.J. Caslake, P. Stewart, A. Soro, J. Westerbacka, B. Wennberg, S.O. Olofsson, M.R. Taskinen, and J. Borén. A new combined multicompartmental model for apolipoprotein B-100 and triglyceride metabolism in VLDL subfractions. Journal of Lipid Research, 46(1):58, 2005.

[3] M. Adiels, M.R. Taskinen, C. Packard, M.J. Caslake, A. Soro-Paavonen, J. Westerbacka, S. Vehkavaara, A. Häkkinen, S.O. Olofsson, and H. Yki-Järvinen. Overproduction of large VLDL particles is driven by increased liver fat content in man. Diabetologia, 49(4):755-765, 2006.

[4] M. Adiels, J. Westerbacka, A. Soro-Paavonen, A.M. Häkkinen, S. Vehkavaara, M.J. Caslake, C. Packard, S.O. Olofsson, H. Yki-Järvinen, and M.R. Taskinen. Acute suppression of VLDL1 secretion rate by insulin is associated with hepatic fat content and insulin resistance. Diabetologia, 50(11):2356-2365, 2007.

[5] V. Andersen, J. Sonne, S. Sletting, and A. Prip. The volume of the liver in patients correlates to body weight and alcohol consumption. Alcohol and Alcoholism, 35(5):531, 2000.

[6] P. Angulo and K.D. Lindor. Non-alcoholic fatty liver disease. Journal of Gastroenterology and Hepatology, 17:S186-S190, 2002.

[7] R.N. Bergman, Y.Z. Ider, C.R. Bowden, and C. Cobelli. Quantitative estimation of insulin sensitivity. American Journal of Physiology-Endocrinology and Metabolism, 236(6):E667-E677, 1979.

[8] A.S.T. Bickerton, R. Roberts, B.A. Fielding, L. Hodson, E.E. Blaak, A.J.M. Wagenmakers, M. Gilbert, F. Karpe, and K.N. Frayn. Preferential uptake of dietary fatty acids in adipose tissue and muscle in the postprandial period. Diabetes, 56(1):168, 2007.

[9] A.S.T. Bickerton, R. Roberts, B.A. Fielding, H. Tornqvist, E.E. Blaak, A.J.M. Wagenmakers, M. Gilbert, S.M. Humphreys, F. Karpe, and K.N. Frayn. Adipose 
tissue fatty acid metabolism in insulin-resistant men. Diabetologia, 51(8):1466$1474,2008$.

[10] R.K. Crane and A. Sols. The non-competitive inhibition of brain hexokinase by glucose-6-phosphate and related compounds. Journal of Biological Chemistry, 210(2):597, 1954.

[11] M.L. Cruz, K. Evans, and K.N. Frayn. Postprandial lipid metabolism and insulin sensitivity in young northern europeans, south asians and latin americans in the UK. Atherosclerosis, 159(2):441-449, 2001.

[12] R.K. Dash, Y. Li, J. Kim, G.M. Saidel, and M.E. Cabrera. Modeling cellular metabolism and energetics in skeletal muscle: large-scale parameter estimation and sensitivity analysis. IEEE Transactions on Biomedical Engineering, 55(4):1298-1318, 2008.

[13] A. De Gaetano and O. Arino. Mathematical modelling of the intravenous glucose tolerance test. Journal of Mathematical Biology, 40(2):136-168, 2000.

[14] K.L. Donnelly, C.I. Smith, S.J. Schwarzenberg, J. Jessurun, M.D. Boldt, E.J. Parks, et al. Sources of fatty acids stored in liver and secreted via lipoproteins in patients with nonalcoholic fatty liver disease. J Clin Invest, 115(5):1343-1351, 2005.

[15] W.C. Duckworth, R.G. Bennett, and F.G. Hamel. Insulin degradation: progress and potential. Endocrine Reviews, 19(5):608, 1998.

[16] J.O. Ebbert and M.D. Jensen. Fat Depots, Free Fatty Acids, and Dyslipidemia Nutrients, 5, 498-508, 2013.

[17] R.V. Farese Jr, , T.J. Yost, and R.H. Eckel. Tissue-specific regulation of lipoprotein lipase activity by insulin/glucose in normal-weight humans. Metabolism, 40(2):214-216, 1991.

[18] F. Fery, N.P. d'Attellis, and E.O. Balasse. Mechanisms of starvation diabetes: a study with double tracer and indirect calorimetry. American Journal of Physiology-Endocrinology and Metabolism, 259(6):E770, 1990. 
[19] K.N. Frayn. Metabolic regulation: a human perspective. WileyBlackwell,Chichester, 2010.

[20] J.M.R. Gill, J.C. Brown, D. Bedford, D.M. Wright, J. Cooney, D.A. Hughes, C.J. Packard, and M.J. Caslake. Hepatic production of VLDL1 but not VLDL2 is related to insulin resistance in normoglycaemic middle-aged subjects. Atherosclerosis, 176(1):49-56, 2004.

[21] S.M. Grundy and H.Y. Mok. Chylomicron clearance in normal and hyperlipidemic man. Metabolism: Clinical and Experimental, 25(11):1225, 1976.

[22] D.E. Harrison, M.R. Christie, and D.W.R. Gray. Properties of isolated human islets of Langerhans: insulin secretion, glucose oxidation and protein phosphorylation. Diabetologia, 28(2):99-103, 1985.

[23] L. Hodson, A.S.T. Bickerton, S.E. McQuaid, R. Roberts, F. Karpe, K.N. Frayn and B.A. Fielding. The contribution of splanchnic fat to VLDL triglyceride is greater in insulin-resistant than insulin-sensitive men and women. Diabetes, 56(10):2433, 2007.

[24] L. Hodson, S.E. McQuaid, S.M. Humphreys, R. Milne, B.A. Fielding, K.N. Frayn, and F. Karpe. Greater dietary fat oxidation in obese compared with lean men: an adaptive mechanism to prevent liver fat accumulation? American Journal of Physiology-Endocrinology and Metabolism, 299(4):E584, 2010.

[25] P.A. Jansson, A. Larsson, U. Smith, and P. Lönnroth. Glycerol production in subcutaneous adipose tissue in lean and obese humans. Journal of Clinical Investigation, 89(5):1610, 1992.

[26] K. Jelic. Biosimulation of Lipid Homeostasis. PhD thesis, Danish Technical University, Lyngby, Denmark, 2003.

[27] K. Jelic, C.E. Hallgreen, and M. Colding-Jørgensen. A model of NEFA dynamics with focus on the postprandial state. Annals of Biomedical Engineering, 37(9):1897-1909, 2009. 
[28] M.D. Jensen, S. Cardin, D. Edgerton, A. Cherrington Splanchnic free fatty acid kinetics. American Journal of Physiology - Endocrinology and Metabolism, 284(6), E1140-E1148, 2003

[29] E.P. Joslin and C.R. Kahn. Joslin's diabetes mellitus. Lippincott Williams \& Wilkins, 2005.

[30] D.E. Kelley, B. Goodpaster, R.R. Wing, and J.A. Simoneau. Skeletal muscle fatty acid metabolism in association with insulin resistance, obesity, and weight loss. American Journal of Physiology-Endocrinology and Metabolism, 277(6):E1130E1141, 1999.

[31] J. Kim. Model analysis of adipose tissue and whole body metabolism in vivo. $\mathrm{PhD}$ thesis, Case Western Reserve University, 2008.

[32] J. Kim, G.M. Saidel, and M.E. Cabrera. Multi-scale computational model of fuel homeostasis during exercise: effect of hormonal control. Annals of Biomedical Engineering, 35(1):69-90, 2007.

[33] J. Kim, G.M. Saidel, and S.C. Kalhan. A computational model of adipose tissue metabolism: evidence for intracellular compartmentation and differential activation of lipases. Journal of Theoretical Biology, 251(3):523-540, 2008.

[34] A. Kotronen, A. Seppälä-Lindroos, S. Vehkavaara, R. Bergholm, K.N. Frayn, B.A. Fielding, and H. Yki-Järvinen. Liver fat and lipid oxidation in humans. Liver International, 29(9):1439-1446, 2009.

[35] E.W. Kraegen, J.A. Sowden, M.B. Halstead, P.W. Clark, K.J. Rodnick, D.J. Chisholm, and D.E. James. Glucose transporters and in vivo glucose uptake in skeletal and cardiac muscle: fasting, insulin stimulation and immunoisolation studies of GLUT1 and GLUT4. Biochemical Journal, 295(Pt 1):287, 1993.

[36] Y. Li, T.P.J. Solomon, J.M. Haus, G.M. Saidel, M.E. Cabrera, and J.P. Kirwan. Computational model of cellular metabolic dynamics: effect of insulin on glucose disposal in human skeletal muscle. American Journal of PhysiologyEndocrinology and Metabolism, 298(6):E1198-E1209, 2010. 
[37] J.E. Liljenquist, J.D. Bomboy, S.B. Lewis, B.C. Sinclair-Smith, P.W. Felts, W.W. Lacy, O.B. Crofford, and G.W. Liddle. Effects of glucagon on lipolysis and ketogenesis in normal and diabetic men. Journal of Clinical Investigation, 53(1):190, 1974 .

[38] R. Malmström, CJ Packard, M. Caslake, D. Bedford, P. Stewart, H. YkiJärvinen, J. Shepherd, and M.R. Taskinen. Defective regulation of triglyceride metabolism by insulin in the liver in NIDDM. Diabetologia, 40(4):454-462, 1997.

[39] R. Malmström, C.J. Packard, M. Caslake, D. Bedford, P. Stewart, H. YkiJärvinen, J. Shepherd, and M.R. Taskinen. Effects of insulin and acipimox on VLDL1 and VLDL2 apolipoprotein B production in normal subjects. Diabetes, 47(5):779, 1998.

[40] CD Man, M. Camilleri, and C. Cobelli. A system model of oral glucose absorption: validation on gold standard data. Biomedical Engineering, IEEE Transactions on, 53(12):2472-2478, 2006.

[41] CD Man, R.A. Rizza, and C. Cobelli. Meal simulation model of the glucoseinsulin system. Biomedical Engineering, IEEE Transactions on, 54(10):17401749, 2007.

[42] A. Mari, A. Tura, A. Gastaldelli, and E. Ferrannini. Assessing insulin secretion by modeling in multiple-meal tests. Diabetes, 51(suppl 1):S221, 2002.

[43] J.D. Murray. Mathematical Bioloy Springer-Verlag, Berlin, 1989.

[44] S Nielsen, ZK Guo, CM Johnson, DD Hensrud and MD Jensen Splanchnic lipolysis in human obesity J Clin Invest. 113(11) 1582-1588 (2004).

[45] R. Occhipinti, MA Puchowicz, JC LaManna, E. Somersalo, and D. Calvetti. Statistical analysis of metabolic pathways of brain metabolism at steady state. Annals of biomedical engineering, 35(6):886-902, 2007.

[46] T. Pearson, J.A.D. Wattis, B. O Malley, L. Pickersgill, H. Blackburn, KG Jackson, and HM Byrne. Mathematical modelling of competitive LDL/VLDL binding and uptake by hepatocytes. Journal of Mathematical Biology, 58(6):845-880, 2009 . 
[47] AC. Pratt. PhD thesis, University of Nottingham, (2014).

[48] T.B. Price, G. Perseghin, A. Duleba, W. Chen, J. Chase, D.L. Rothman, R.G. Shulman, and G.I. Shulman. NMR studies of muscle glycogen synthesis in insulinresistant offspring of parents with non-insulin-dependent diabetes mellitus immediately after glycogen-depleting exercise. Proceedings of the National Academy of Sciences, 93(11):5329, 1996.

[49] V.T. Samuel, Z.X. Liu, X. Qu, B.D. Elder, S. Bilz, D. Befroy, A.J. Romanelli, and G.I. Shulman. Mechanism of hepatic insulin resistance in non-alcoholic fatty liver disease. Journal of Biological Chemistry, 279(31):32345, 2004.

[50] P.R. Shorten and G.C. Upreti. A mathematical model of fatty acid metabolism and vldl assembly in human liver. Biochimica et Biophysica Acta (BBA)Molecular and Cell Biology of Lipids, 1736(2):94-108, 2005.

[51] G.I. Shulman, D.L. Rothman, T. Jue, P. Stein, R.A. DeFronzo, and R.G. Shulman. Quantitation of muscle glycogen synthesis in normal subjects and subjects with non-insulin-dependent diabetes by $13 \mathrm{C}$ nuclear magnetic resonance spectroscopy. New England Journal of Medicine, 322(4):223-228, 1990.

[52] E Søndergaard, LP Sørensen, I Rahbek, LC Gormsen, JS Christiansen, S Nielsen. Postprandial VLDL-triacylglycerol secretion is not suppressed in obese type 2 diabetic men. Diabetologia, 55, 2733-2740, (2012).

[53] S.K. Sreenan, B.N. Cockburn, A.C. Baldwin, D.M. Ostrega, M. Levisetti, A. Grupe, G.I. Bell, T.A. Stewart, M.W. Roe, and K.S. Polonsky. Adaptation to hyperglycemia enhances insulin secretion in glucokinase mutant mice. Diabetes, 47(12):1881, 1998.

[54] J. Sturis, K.S. Polonsky, E. Mosekilde, and E. Van Cauter. Computer model for mechanisms underlying ultradian oscillations of insulin and glucose. American Journal of Physiology-Endocrinology and Metabolism, 260(5):E801-E809, 1991.

[55] R. Taylor, I. Magnusson, D.L. Rothman, G.W. Cline, A. Caumo, C. Cobelli, and G.I. Shulman. Direct assessment of liver glycogen storage by $13 \mathrm{C}$ nuclear magnetic resonance spectroscopy and regulation of glucose homeostasis after a 
mixed meal in normal subjects. Journal of Clinical Investigation, 97(1):126, 1996.

[56] R.G. Tiessen, M.M. Rhemrev-Boom, and J. Korf. Glucose gradient differences in subcutaneous tissue of healthy volunteers assessed with ultraslow microdialysis and a nanolitre glucose sensor. Life Sciences, 70(21):2457-2466, 2002.

[57] M.T. Timlin and E.J. Parks. Temporal pattern of de novo lipogenesis in the postprandial state in healthy men. American Journal of Clinical Nutrition, 81(1):35, 2005.

[58] M.J. Tindall, J.A.D. Wattis, B.J. O’Malley, L. Pickersgill, and K.G. Jackson. A continuum receptor model of hepatic lipoprotein metabolism. Journal of Theoretical Biology, 257(3):371-384, 2009.

[59] John Wahren and Karin Ekberg. Splanchnic regulation of glucose production. Annu. Rev. Nutr., 27:329-345, 2007.

[60] J. Westerbacka, A. Kotronen, B.A. Fielding, J. Wahren, L. Hodson, J. Perttilä, T. Seppänen-Laakso, T. Suortti, J. Arola, and R. Hultcrantz. Splanchnic balance of free fatty acids, endocannabinoids, and lipids in subjects with nonalcoholic fatty liver disease. Gastroenterology, 139(6):1961-1971, 2010. 